\title{
Objective magnetic vortex detection
}

\author{
Erico L. Rempel,,${ }^{1,2, *}$ Tiago F. P. Gomes, ${ }^{2}$ Suzana S. A. Silva, ${ }^{1}$ and Abraham C.-L. Chian ${ }^{1,2,3}$ \\ ${ }^{1}$ Institute of Aeronautical Technology (ITA), World Institute for Space Environment Research (WISER), \\ São José dos Campos, São Paulo 12228-900, Brazil \\ ${ }^{2}$ National Institute for Space Research (INPE), P.O. Box 515, São José dos Campos, São Paulo 12227-010, Brazil \\ ${ }^{3}$ University of Adelaide, School of Mathematical Sciences, Adelaide, South Australia 5005, Australia
}

(Received 7 November 2018; revised manuscript received 25 February 2019; published 29 April 2019)

\begin{abstract}
Magnetic coherent vortical structures are ubiquitous in space and astrophysical plasmas and their detection is key to understanding the nature of the intrinsic turbulence in those conducting fluids. A recently developed method to detect magnetic vortices is explored in problems of two- and three-dimensional magnetohydrodynamic simulations. The integrated averaged current deviation, the normed difference of the current density at a point and the mean current density in the domain, integrated along a magnetic field line, is proved to be objective, i.e., invariant under rotations and translations of the observer. The method is shown to detect accurately the boundary of magnetic vortices in two-dimensional simulations, as well as magnetic flux ropes in three dimensions.
\end{abstract}

DOI: 10.1103/PhysRevE.99.043206

\section{INTRODUCTION}

Vortices are the natural building blocks of turbulence, since the nonlinear energy cascade where large vortices break up into smaller vortices is thought to be responsible for the scaling laws found in kinetic energy spectra in fully developed turbulence [1]. These persistent coherent structures that enclose regions with high vorticity have been studied for decades in hydrodynamical problems, but there is still no standard definition of what a vortex is or how it can be detected (see, e.g., Haller et al. [2] and references therein). In the context of space and astrophysical plasmas, it is usually imperative to consider the presence of magnetic fields, with their own vortical structures. In two spatial dimensions, these structures exhibit a curling in-plane magnetic configuration, with field lines encircling or spiraling around a center. Kinney et al. [3] formally defined magnetic vortices as axisymmetric monopole patterns in the current field. In three dimensions, magnetic vortices constitute a bundle of helical field lines in a tubular region. This work focuses on frame invariant detection of two-dimensional (2D) and three-dimensional (3D) magnetic vortices in magnetohydrodynamic (MHD) simulations.

A number of papers have studied current sheets, magnetic islands, vortices, and magnetic reconnection in twodimensional incompressible MHD turbulence. For example, Orszag and Tang [4] introduced a two-dimensional prototype model for numerical simulation of small-scale structures (singularities) of vortices and current sheets in an incompressible MHD flow, and showed that 2D MHD turbulence is less singular (containing less small-scale structures) than 3D hydrodynamic turbulence, but more singular than 2D hydrodynamic turbulence. Biskamp and Welter [5] investigated the dynamics of 2D decaying incompressible MHD turbulence using two types of initial states: (i) a generalization of the initial

\footnotetext{
*rempel@ita.br
}

conditions of [4] to facilitate the generation of small-scale structures, and (ii) an initial state having dominant scales that are small compared with the system size, defined by a broad spectrum of modes with random phases, to allow an inverse cascade to develop. They obtained a turbulent power spectrum that exhibits both Kraichnan and Kolmogorov spectral indices in the inertial range and a modified dissipation scale driven by intermittency in the dissipation range. Politano et al. [6] performed high-resolution simulations of the Orszag-Tang model of MHD turbulence at Reynolds numbers of several thousand, yielding kinetic and magnetic energy spectra that display an inertial range extending over one decade of wave numbers, and a dissipation range where resistive tearing instabilities destabilize current sheets generated by the inertial dynamics, leading to the formation of small-scale magnetic islands. They tested the generality of their results of the Orszag-Tang vortex by considering flows with random initial conditions. Wu and Chang [7] reported the onset and interactions of localized coherent structures in two-dimensional MHD simulations. They noticed that the structures can merge locally and sporadically in the absence of a background magnetic field. When there is a background sheared magnetic field, the structures tend to align with the background current sheet, a process that was related to an intermittent turbulence model for the Earth's magnetotail. Armstrong and Craig [8,9] studied viscous and resistive losses in transient magnetic reconnection driven by the Orszag-Tang vortex and concluded that viscous dissipation in solar flaring plasmas can account for a significant fraction of the flare energy release.

In three spatial dimensions, magnetic vortices are often known as flux ropes [10]. Inside these tubular or cylindrical regions with spiraling or helical magnetic field lines, the axial magnetic field is much larger than the magnetic field outside. However, this definition does not provide a precise description of the vortex boundary, since a user-defined magnetic threshold is required to determine whether field lines are considered inside or outside the rope. Hence, several works 
on flux tubes or ropes display those structures by plotting the isosurfaces of the magnetic field or current density, where the user chooses some energy level to define the surface to be plotted. For example, in a 3D data set assembled using the relaxation scaling experiment device, Sears et al. [11] detected the signature of flux ropes transversal to a horizontal plane as regions where the contours of flux rope current density $\boldsymbol{J}_{z}(x, y)$ fall below a certain threshold. Isosurfaces and contour plots of different quantities related to the parallel current and helical magnetic field components were used to locate flux ropes in MHD simulations of the Pegasus Toroidal Experiment in [12]. In [13] the thresholding is applied to the magnetic helicity mapping, a quantity defined as the magnetic helicity normalized by the magnitude of the magnetic field, integrated along the field lines. The difficulty in defining vortex boundaries also occurs in two dimensions, where the vortex centers can be identified as local extrema of the current [3], but their boundaries must be inferred in some way from contour plots.

Rempel et al. [14] proposed a new and simple way to define magnetic vortices based on the integrated averaged current deviation (IACD), which is the integral of the normed difference between the current density along a magnetic field line and the current density averaged in the spatial domain. The vortex boundary is defined as the outermost convex contour surrounding a local maximum of the IACD field. The method is based on an adaptation of the Lagrangian averaged vorticity deviation (LAVD), introduced by Haller et al. [2] for kinematic vortices and recently employed in the detection of coherent swirls in an ocean general circulation model simulation of the Gulf of Mexico [15]. A few preliminary results on IACD were shown in [14] using numerical simulations of a nonlinear dynamo. In the present paper, we apply the IACD to magnetic vortex detection in a series of two- and threedimensional MHD simulations to test its robustness in different circumstances, both in compressible and incompressible flows. The IACD method is shown to be effective in detecting vortex boundaries in which the magnetic field lines spiral together without reconnecting and spreading away. In three dimensions, the field lines from boundaries found on a plane section keep their coherence in a tubular region, showing that the method can be employed to detect flux ropes. Possible applications in the context of solar plasmas are discussed. We also prove that IACD vortices are objective, which means that they remain unchanged under translations and rotations of the coordinate frame. This point is fundamental in a definition of a magnetic vortex, and is a hallmark of the IACD method. If a detection method is not objective it means that a perfectly straight magnetic field line could be classified as a twisted line when measured with respect to a rotating frame (see Fig. 5 in [16]).

In Sec. II, we discuss the important concept of objectivity, which guarantees that an operator is invariant under continuous changes of an observer. The concept is applied to the LAVD and IACD operators. A precise definition of a magnetic vortex is provided. Section III contains a detailed proof of the objectivity of IACD. Section IV presents the results of MHD simulations in three situations: two-dimensional incompressible simulations with Orszag-Tang vortices as initial conditions, two-dimensional incompressible simulations with random vortices as initial conditions, and three-dimensional compressible simulations of interlocked flux rings as initial conditions. Section V gives the conclusions.

\section{OBJECTIVE KINEMATIC AND MAGNETIC VORTICES}

\section{A. Objectivity}

The concept of objectivity refers to invariance under time-dependent transformations of a reference frame or observer [17]. The invariance of a quantity is important in the context of vortex identification because a detection technique must be able to correctly classify a flow region as a vortex independent of the observer. The topic has been extensively discussed in the literature (see, e.g., [18]).

The invariance of a physical quantity is also important when there is no time evolution involved, i.e., in steady flows. For example, the description of coherent structures in an instantaneous magnetic field might depend on the frame used, since an observer $\mathcal{O}$ translated and rotated in relation to another observer $\overline{\mathcal{O}}$ may see the magnetic field lines with a different topology. As an example, suppose that observer $\mathcal{O}$ measures the following magnetic field at time $t_{0}$ :

$$
\boldsymbol{B}(\boldsymbol{x})=\left(\begin{array}{lll}
0 & 1 & 0 \\
1 & 0 & 0 \\
0 & 0 & 1
\end{array}\right) \boldsymbol{x}, \quad \boldsymbol{x} \in \mathbb{R}^{3} .
$$

The magnetic field lines observed by $\mathcal{O}$ at time $t_{0}$ are shown in Fig. 1(a) and reveal diverging lines surrounding a saddle fixed point in the middle of the box. The field lines are solutions of the initial value problem

$$
\frac{d \boldsymbol{x}}{d s}=\boldsymbol{B}(\boldsymbol{x}(s)), \quad \boldsymbol{x}\left(s_{0}\right)=\boldsymbol{x}_{0},
$$

where $\boldsymbol{x}(s)$ is the position vector and $s$ is a scalar parameter related to the distance $l$ along the field line by $d l=|\boldsymbol{B}| d s$.

Next, suppose that observer $\overline{\mathcal{O}}$ is rotated and translated in relation to $\mathcal{O}$, such that position vectors are related by the following Euclidean transformation:

$$
\overline{\boldsymbol{x}}=\boldsymbol{c}(s)+\mathbf{Q}(s) \boldsymbol{x}, \quad \bar{s}=s+a,
$$

where $\mathbf{Q}(s)$ is an arbitrary orthogonal rotation matrix, $\boldsymbol{c}(s)$ is a translation vector, and $a$ is an arbitrary real constant. Following Eq. (2), the magnetic field observed by $\overline{\mathcal{O}}$ can be obtained by deriving Eq. (3):

$$
\begin{aligned}
\overline{\boldsymbol{B}} & =\frac{d \overline{\boldsymbol{x}}}{d s}=\frac{d \boldsymbol{c}}{d s}+\frac{d \boldsymbol{Q}}{d s} \boldsymbol{x}+\boldsymbol{Q} \frac{d \boldsymbol{x}}{d s} \\
& =\boldsymbol{c}^{\prime}+\boldsymbol{Q}^{\prime} \boldsymbol{x}+\boldsymbol{Q B} \\
& =\boldsymbol{c}^{\prime}+\boldsymbol{Q}^{\prime} \boldsymbol{Q}^{\top}(\overline{\boldsymbol{x}}-\boldsymbol{c})+\boldsymbol{Q B}
\end{aligned}
$$

where ${ }^{\top}$ denotes the transpose operator.

Now, suppose that $\boldsymbol{Q}$ and $\boldsymbol{c}$ are given by

$$
\mathbf{Q}=\left(\begin{array}{ccc}
\cos (s) & 0 & \sin (s) \\
0 & 1 & 0 \\
-\sin (s) & 0 & \cos (s)
\end{array}\right), \quad \boldsymbol{c}=\left(\begin{array}{l}
0 \\
0 \\
0
\end{array}\right) .
$$

Then, for $s=2.1$, the field lines computed from $\overline{\boldsymbol{B}}$ as observed by $\overline{\mathcal{O}}$ behave as in Fig. 1(b). The field lines reveal swirling patterns that could be classified as a magnetic vortex. This 
(a)

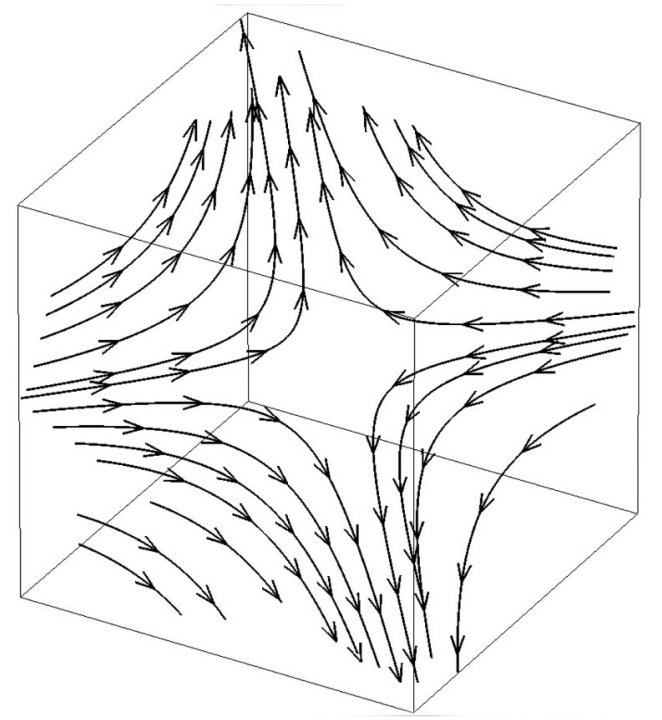

(b)

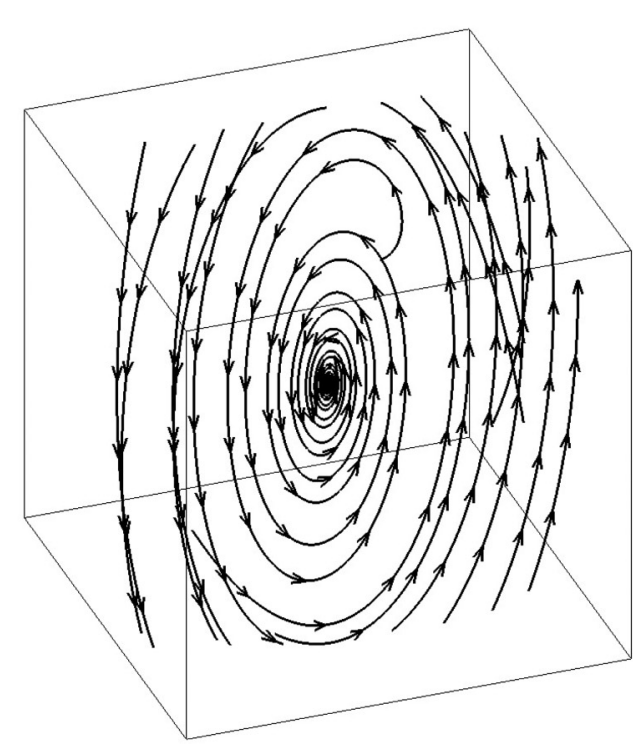

FIG. 1. Magnetic field lines computed from a vector field measured by two different observers: (a) an observer $\mathcal{O}$ detects a saddle point structure and (b) another observer $\overline{\mathcal{O}}$ rotated and translated in relation to $\mathcal{O}$ detects a magnetic vortex.

shows that magnetic field lines are not an objective quantity, since the same magnetic structure could be classified as either a saddle point or a vortex core, depending on the observer. A practical situation is the study of magnetic structures in space and solar plasmas as observed by different satellites at a given time. One would like them both to agree on the kind of structure observed, and correcting the orientation of one satellite to match the other does not solve the problem, since they could both accuse the detection of a vortex when in fact they are observing a rotated saddle structure. An example similar to the one in Fig. 1 is provided by [19] in the context of continuous rotations of a frame in two-dimensional timedependent velocity fields.

The problem described above is essentially the same as computing the streamlines traced by a passive scalar in a steady velocity field as $t$ evolves. In the case of magnetic field lines, the line parameter $s$ is used in place of $t$, and one can discuss objectivity in terms of position and $s$. Suppose that an event is characterized by observers $\mathcal{O}$ and $\overline{\mathcal{O}}$, respectively, by $(\boldsymbol{x}, s)$ and $(\overline{\boldsymbol{x}}, \bar{s})$, which are related by a Euclidean transformation as in Eq. (3). Then, a point difference vector $v$ is defined as objective or frame indifferent if it conserves its magnitude in different frames; thus, $v$ is objective if it transforms as

$$
\bar{v}=\mathbf{Q}(s) v,
$$

since the rotation matrix preserves the length of a vector. Also, in the context of steady fields, a tensor is said to be objective if

$$
\overline{\mathbf{T}}=\mathbf{Q}(s) \mathbf{T Q}(s)^{\top} .
$$

Equations (3), (6), and (7) can be applied when describing the properties of a steady flow or a magnetic vector field at a given time.

\section{B. Objective vortex detection}

\section{Lagrangian averaged vorticity deviation}

Most of the existing methods for defining or detecting vortices are not objective. Haller et al. [2] developed a method to define coherent kinematic vortices objectively. Consider a velocity field $\boldsymbol{u}$ in a spatial domain $U$. A fluid particle at initial position $\boldsymbol{x}\left(t_{0}\right)=\boldsymbol{x}_{0} \in U$ follows a trajectory in space given by solutions of the initial value problem

$$
\frac{d \boldsymbol{x}}{d t}=\boldsymbol{u}(\boldsymbol{x}, t), \quad \boldsymbol{x}\left(t_{0}\right)=\boldsymbol{x}_{0} .
$$

The Lagrangian averaged vorticity deviation is defined as

$$
\operatorname{LAVD}_{t_{0}}^{t_{0}+\tau}\left(x_{0}\right)=\int_{t_{0}}^{t_{0}+\tau}|\omega(x(t), t)-\langle\omega(t)\rangle| d t,
$$

where $\tau$ is a given time interval related to the typical lifetime of the vortices that are to be found, $\omega=\nabla \times \boldsymbol{u}$ is the vorticity, and $\langle\omega(t)\rangle$ is the spatial mean of the vorticity at time $t$. An objective kinematic Lagrangian vortex is, then, defined as an evolving convex material domain $\mathcal{V}_{L}(t)$ such that $\mathcal{V}_{L}\left(t_{0}\right)$ is filled with a set of level curves (for twodimensional flows) or surfaces (for three-dimensional flows) of $\operatorname{LAVD}_{t_{0}}^{t_{0}+\tau}(\boldsymbol{x})$ with outward nonincreasing LAVD values. In practice, one can search for kinematic Lagrangian vortices in a two-dimensional flow by computing the LAVD field on the plane, extracting the initial positions of vortices as the local maxima of LAVD, and then computing a vortex boundary as the outermost convex closed contour of LAVD surrounding a vortex center, i.e., a local LAVD maximum. In three-dimensional flows, the above procedure can be used to find the intersection of a vortex with a plane in the flow domain.

In order to take into account small deviations from convexity that are always present in data with finite resolution, the convexity deficiency was introduced by [2]

$$
\varepsilon:=\frac{A_{c}-A_{c h}}{A_{c}}
$$

where $A_{c}$ is the area enclosed by the contour extracted and $A_{c h}$ is the area enclosed by its convex hull. Thus, an outermost closed contour is classified as a vortex boundary if $\varepsilon$ is less than a given threshold. The threshold value is problem 
dependent and must be chosen after some trial and error. It must be small enough to avoid classifying the numerical approximation of nonconvex structures as convex and large enough to account for the ragged edges resulting from the discretization of the LAVD field. Previous experiments with observational and simulated data in two and three dimensions using a LAVD field with $512^{2}$ [2] or $512^{3}$ [14] grid points revealed good results using $\varepsilon$ between $10^{-3}$ and $10^{-5}$.

\section{Integrated averaged current deviation}

In order to detect magnetic vortices, Rempel et al. [14] introduced a variation of the LAVD technique based on the magnetic field and the current density. Consider the magnetic field line equation [Eq. (2)] at $t=t_{0}$. Then, the IACD is defined as

$$
\operatorname{IACD}_{s_{0}}^{s_{0}+\xi}\left(\boldsymbol{x}_{0}\right)=\int_{s_{0}}^{s_{0}+\xi}\left|\boldsymbol{J}\left(\boldsymbol{x}(s), t_{0}\right)-\left\langle\boldsymbol{J}\left(t_{0}\right)\right\rangle\right| d s,
$$

where $\boldsymbol{J}=\nabla \times \boldsymbol{B} / \mu_{0}$ is the current density and $\left\langle\boldsymbol{J}\left(t_{0}\right)\right\rangle$ is the mean current density. Note that, differently from LAVD, the calculations of IACD are performed for a fixed time $t_{0}$, due to the fact that the field line equation, Eq. (2), is defined for a fixed time. The IACD is an objective quantity, which guarantees its invariance under arbitrary, continually varying observer changes along magnetic lines. Thus, given arbitrary rotation matrix $\mathbf{Q}(s)$ and translation vector $\boldsymbol{c}(s)$, if $\overline{\boldsymbol{J}}=\mathbf{Q}(s) \boldsymbol{J}+\boldsymbol{c}(s)$, then

$$
|\overline{\boldsymbol{J}}-\langle\overline{\boldsymbol{J}}\rangle|=|\boldsymbol{J}-\langle\boldsymbol{J}\rangle|,
$$

as demonstrated in Sec. III.

For a given parameter interval $\left[s_{0}, s_{0}+\xi\right]$, a magnetic vortex is defined as a convex domain $\mathcal{V}_{L}\left(t_{0}\right)$ such that $\mathcal{V}_{L}\left(t_{0}\right)$ is filled with a set of tubular level curves or surfaces of $\operatorname{IACD}_{s_{0}}^{s_{0}+\xi}\left(\boldsymbol{x}_{0}\right)$ with outward nonincreasing IACD values; the boundary of $\mathcal{V}_{L}\left(t_{0}\right)$ is the outermost convex level curve or surface of $\operatorname{IACD}_{s_{0}}^{s_{0}+\xi}\left(\boldsymbol{x}_{0}\right)$ in $\mathcal{V}_{L}\left(t_{0}\right)$. The numerical computation of magnetic vortices in two- and three-dimensional fields follows the same procedure described above for objective kinematic vortices, just changing LAVD by IACD.

The parameter $\xi$ determines the length along the field lines inside the structure to be considered as a coherent magnetic vortex and is usually chosen after some trial and error, depending on each problem. In three-dimensional systems, $\xi$ is related to the length of magnetic flux ropes, so larger values of $\xi$ should be used to detect longer ropes, which will typically be thinner than the ropes found with smaller $\xi$, as illustrated in Sec. IV B.

Solving Eq. (2) requires integrating field lines in a continuous space domain. Since we are working with discrete data for $\boldsymbol{B}$ obtained from numerical simulations of the MHD equations, we use cubic splines interpolation for the $\boldsymbol{B}$ field and solve Eq. (2) with a fourth-order Runge-Kutta integrator.

\section{PROOF OF THE OBJECTIVITY OF IACD}

The objectivity of LAVD was demonstrated in [2] using the velocity gradient tensor and the definition of the spin tensor. We follow a similar approach for IACD based on the magnetic gradient and current density tensors. The magnetic gradient

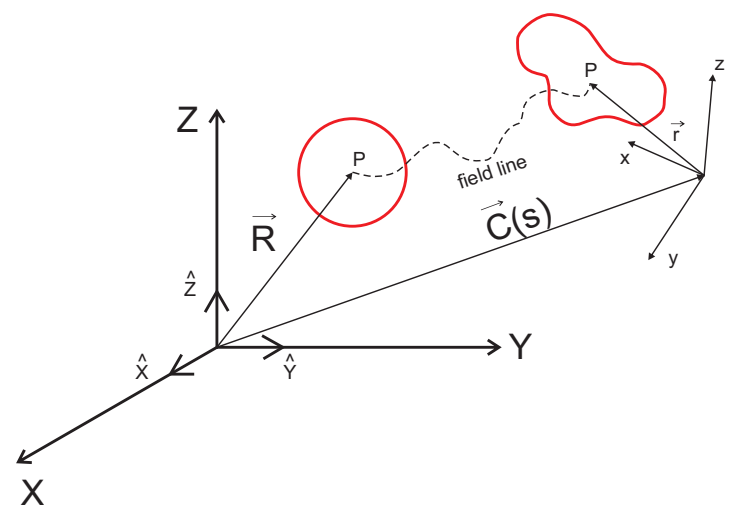

FIG. 2. "Lagrangian" and spatial coordinates of a deformable body.

tensor components can be written as [20]

$$
\nabla \boldsymbol{B}=\left(\begin{array}{lll}
\partial_{x} B_{x} & \partial_{y} B_{x} & \partial_{z} B_{x} \\
\partial_{x} B_{y} & \partial_{y} B_{y} & \partial_{z} B_{y} \\
\partial_{x} B_{z} & \partial_{y} B_{z} & \partial_{z} B_{z}
\end{array}\right) .
$$

The magnetic gradient tensor can be written in terms of its symmetric (S) and antisymmetric (A) parts,

$$
\nabla \boldsymbol{B}=\mathbf{S}+\mathbf{A},
$$

where

$$
\begin{aligned}
& \mathbf{S}=\frac{\nabla \boldsymbol{B}+\nabla \boldsymbol{B}^{\top}}{2}, \\
& \mathbf{A}=\frac{\nabla \boldsymbol{B}-\nabla \boldsymbol{B}^{\top}}{2} .
\end{aligned}
$$

Substituting the components of $\nabla \boldsymbol{B}$ in $\mathbf{A}$, we obtain the following matrix:

$$
\mathbf{A}=\frac{1}{2}\left(\begin{array}{ccc}
0 & \partial_{y} B_{x}-\partial_{x} B_{y} & \partial_{z} B_{x}-\partial_{x} B_{z} \\
\partial_{x} B_{y}-\partial_{y} B_{x} & 0 & \partial_{z} B_{y}-\partial_{y} B_{z} \\
\partial_{x} B_{z}-\partial_{z} B_{x} & \partial_{y} B_{z}-\partial_{z} B_{y} & 0
\end{array}\right) .
$$

From Ampère's law,

$$
\begin{aligned}
\nabla \times \boldsymbol{B}= & \left(\partial_{y} B_{z}-\partial_{z} B_{y}\right) \hat{x}+\left(\partial_{z} B_{x}-\partial_{x} B_{z}\right) \hat{y} \\
& +\left(\partial_{x} B_{y}-\partial_{y} B_{x}\right) \hat{z}=\mu_{0} \boldsymbol{J} .
\end{aligned}
$$

Thus, matrix A can be written as

$$
\mathbf{A}=\frac{\mu_{0}}{2}\left(\begin{array}{ccc}
0 & -J_{z} & J_{y} \\
J_{z} & 0 & -J_{x} \\
-J_{y} & J_{x} & 0
\end{array}\right) \Rightarrow \mathbf{A}=\frac{\mu_{0}}{2} \mathbf{J},
$$

where $\mathbf{J}$ is defined as the current density tensor [20]. This tensor has the property that $\mathbf{J}=-\mathbf{J}^{\top}$.

Now, let $\boldsymbol{r}=\boldsymbol{r}(\boldsymbol{R}, s)$ be a spatial vector position and $\boldsymbol{R}=$ $\boldsymbol{R}(\boldsymbol{r}, s)$ the associated material vector position, where $s$ is a parameter. The components of $\boldsymbol{r}$ and $\boldsymbol{R}$ provide the "Lagrangian" and spatial coordinates, respectively. Figure 2 illustrates the Eulerian and Lagrangian position vectors of a point $\mathrm{P}$ in a 


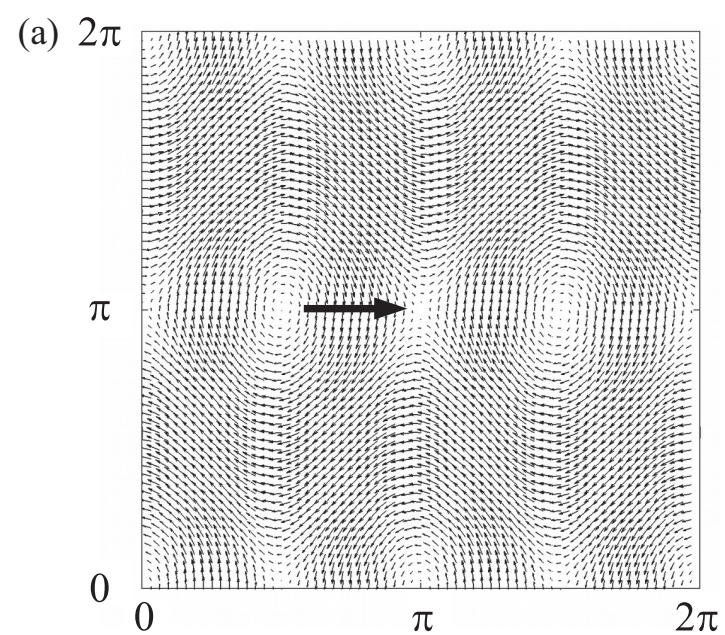

(b)

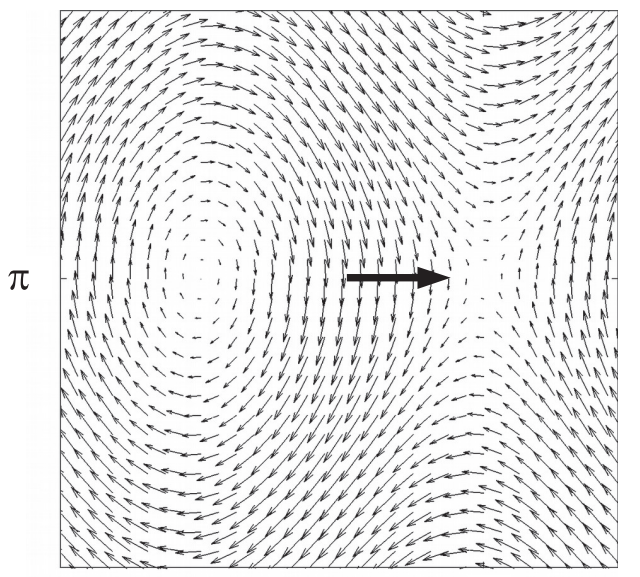

(c)

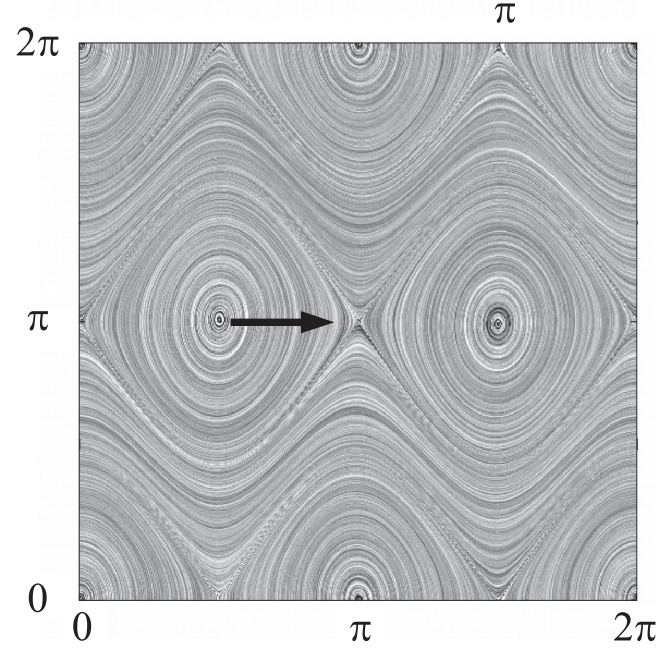

FIG. 3. Orszag-Tang vortices at $t_{0}$ : (a) the magnetic field vectors, (b) an enlargement of the central region of (a), and (c) line integral convolution plot showing the magnetic field lines. The large arrows indicate the location of an $X$ point.

body represented by the red line. In a frame following the field line parametrized by $s$ (the "Lagrangian" frame), the material coordinates are fixed for a given $s$.

The material coordinates are used to define the undeformed reference configuration and the spatial coordinates define the deformed current configuration. In this sense, the Lagrangian
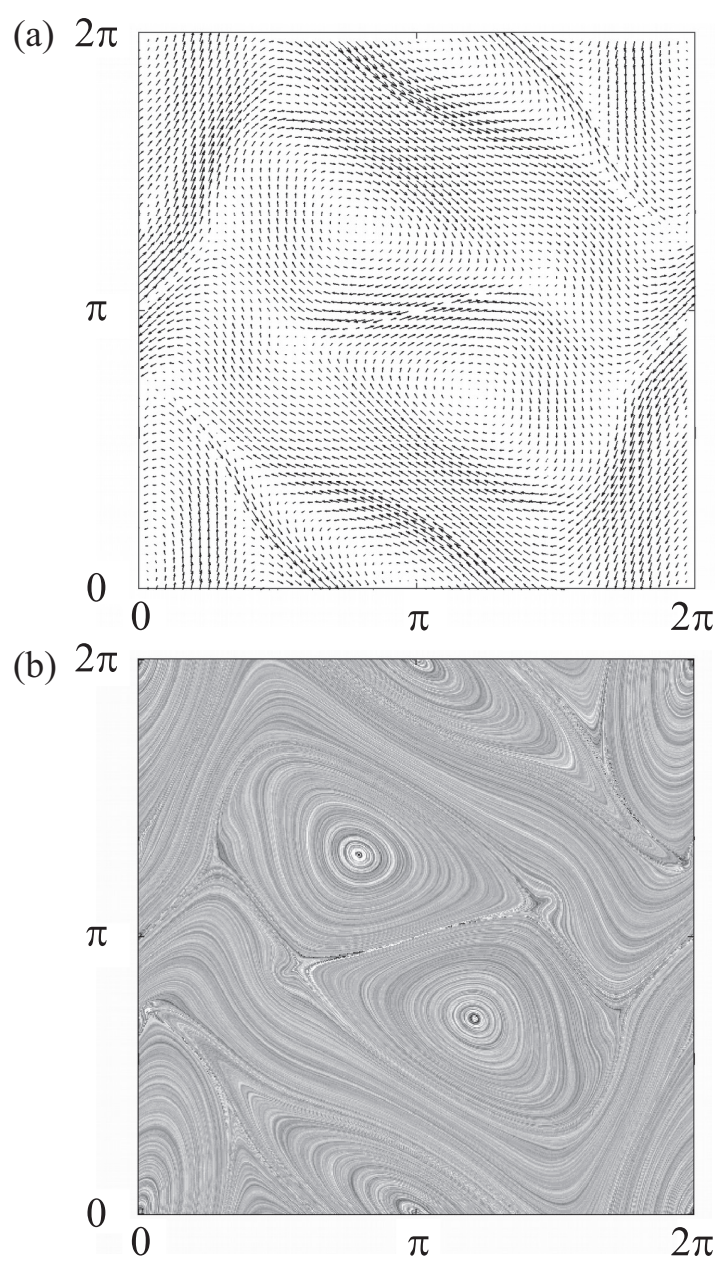

FIG. 4. Orszag-Tang vortices at $t=1$ : (a) the magnetic field vectors and (b) line integral convolution plot showing the magnetic field lines.

description of the deformation gradient tensor is given by

$$
\mathbf{F}=\frac{\partial \boldsymbol{r}}{\partial \boldsymbol{R}}=\left(\begin{array}{lll}
\frac{\partial x}{\partial X} & \frac{\partial x}{\partial Y} & \frac{\partial x}{\partial Z} \\
\frac{\partial y}{\partial X} & \frac{\partial y}{\partial Y} & \frac{\partial y}{\partial Z} \\
\frac{\partial z}{\partial X} & \frac{\partial z}{\partial Y} & \frac{\partial z}{\partial Z}
\end{array}\right) .
$$

The transformed deformation gradient tensor is given by

$$
\overline{\mathbf{F}}=\frac{\partial \overline{\boldsymbol{r}}}{\partial \boldsymbol{R}}=\frac{\partial(\mathbf{Q}(s) \boldsymbol{r}+\boldsymbol{c}(s))}{\partial \boldsymbol{R}}=\mathbf{Q} \frac{\partial \boldsymbol{r}}{\partial \boldsymbol{R}} \Rightarrow \overline{\mathbf{F}}=\mathbf{Q F} .
$$

The derivative of the deformation gradient tensor with respect to the parameter $s$ is defined by

$$
\mathbf{F}^{\prime}=\frac{\partial \mathbf{F}}{\partial s}=\frac{\partial}{\partial s}\left(\frac{\partial \boldsymbol{r}}{\partial \boldsymbol{R}}\right)=\frac{\partial}{\partial \boldsymbol{R}}\left(\frac{\partial \boldsymbol{r}}{\partial s}\right) \Rightarrow \mathbf{F}^{\prime}=\frac{\partial \boldsymbol{B}}{\partial \boldsymbol{R}},
$$

where we used the field line equation $\left(\frac{\partial r}{\partial s}=\boldsymbol{B}\right)$ and the fact that the material coordinate $\boldsymbol{R}$ does not depend on the parameter $s$. From Eq. (21), we can write the magnetic gradient tensor in terms of the deformation gradient tensor as

$$
\mathbf{F}^{\prime}=\frac{\partial \boldsymbol{B}}{\partial \boldsymbol{R}}=\frac{\partial \boldsymbol{B}}{\partial \boldsymbol{r}} \frac{\partial \boldsymbol{r}}{\partial \boldsymbol{R}} \Rightarrow \mathbf{F}^{\prime}=\nabla \boldsymbol{B} \mathbf{F} \Rightarrow \nabla \boldsymbol{B}=\mathbf{F}^{\prime} \mathbf{F}^{-1} \text {. }
$$


(a) $2 \pi$

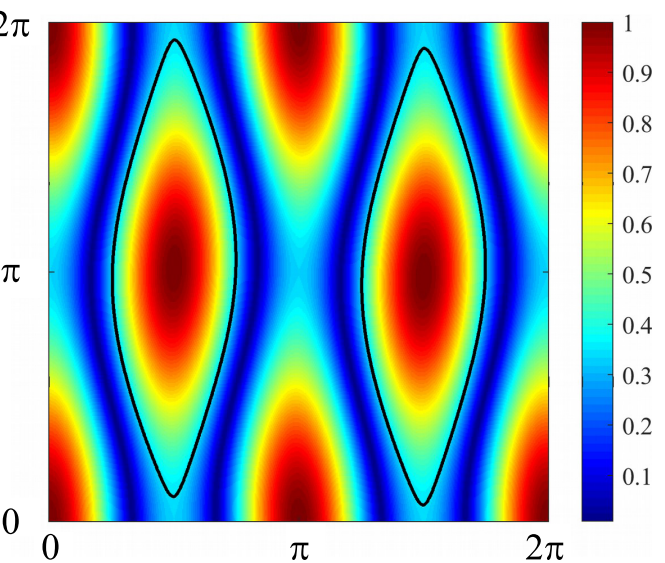

(b)

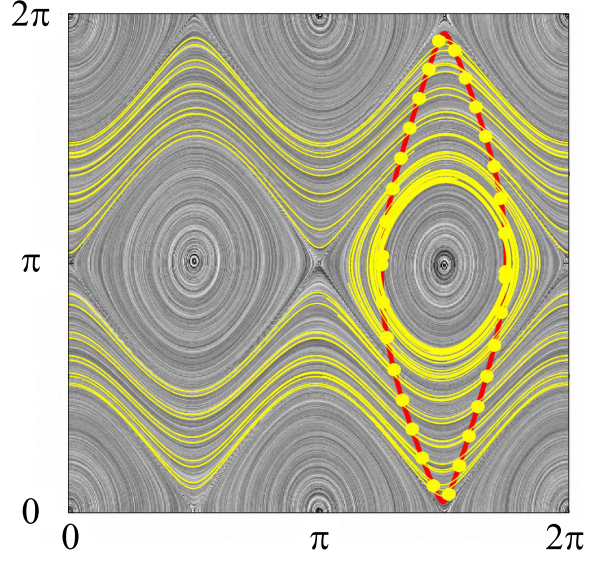

FIG. 5. Orszag-Tang current density at $t_{0}$ : (a) the normalized magnitude of the current density and the vortices detected as convex closed curves (black) from the contour plot of $|\boldsymbol{J}|$ and (b) the line integral convolution plot of the magnetic field lines (dark gray) and the field lines starting from initial conditions (light gray [yellow online] circles) on the $\boldsymbol{J}$ vortex boundary shown in (a) (red).

Using Eqs. (20) and (22) we can show that the magnetic gradient tensor is not objective,

$$
\begin{aligned}
\bar{\nabla} \overline{\boldsymbol{B}} & =\overline{\mathbf{F}}^{\prime} \overline{\mathbf{F}}^{-1}=(\mathbf{Q F})^{\prime}(\mathbf{Q F})^{-1} \\
& =\left(\mathbf{Q}^{\prime} \mathbf{F}+\mathbf{Q} \mathbf{F}^{\prime}\right)\left(\mathbf{F}^{-1} \mathbf{Q}^{-1}\right) \\
& =\mathbf{Q}^{\prime} \mathbf{F} \mathbf{F}^{-1} \mathbf{Q}^{\top}+\mathbf{Q F}^{\prime} \mathbf{F}^{-1} \mathbf{Q}^{\top} \\
& =\mathbf{Q}^{\prime} \mathbf{Q}^{\top}+\mathbf{Q} \nabla \boldsymbol{B} \mathbf{Q}^{\top},
\end{aligned}
$$

where the fact that $\mathbf{Q}$ is an orthogonal matrix was used $\left(\mathbf{Q}^{\top}=\mathbf{Q}^{-1}\right)$.

We can use the results from Eq. (23) to prove that the antisymmetrical part of $\nabla \mathbf{B}$, Eq. (15), is not objective either:

$$
\begin{aligned}
\overline{\mathbf{A}} & =\frac{\bar{\nabla} \overline{\boldsymbol{B}}-\bar{\nabla} \overline{\boldsymbol{B}}^{\top}}{2} \\
& =\frac{1}{2}\left[\mathbf{Q}^{\prime} \mathbf{Q}^{\top}+\mathbf{Q} \nabla \boldsymbol{B} \mathbf{Q}^{\top}-\left(\mathbf{Q}^{\prime} \mathbf{Q}^{\top}+\mathbf{Q} \nabla \boldsymbol{B} \mathbf{Q}^{\top}\right)^{\top}\right] \\
& =\frac{1}{2}\left[\mathbf{Q}^{\prime} \mathbf{Q}^{\top}+\mathbf{Q} \nabla \boldsymbol{B} \mathbf{Q}^{\top}-\mathbf{Q} \mathbf{Q}^{\prime}-\mathbf{Q} \nabla \boldsymbol{B}^{\top} \mathbf{Q}^{\top}\right] \\
& =\frac{1}{2}\left[\mathbf{Q}\left(\nabla \boldsymbol{B}-\nabla \boldsymbol{B}^{\top}\right) \mathbf{Q}^{\top}+2 \mathbf{Q}^{\prime} \mathbf{Q}^{\top}\right] \\
\overline{\mathbf{A}} & =\mathbf{Q} \mathbf{A} \mathbf{Q}^{\top}+\mathbf{Q}^{\prime} \mathbf{Q}^{\top},
\end{aligned}
$$

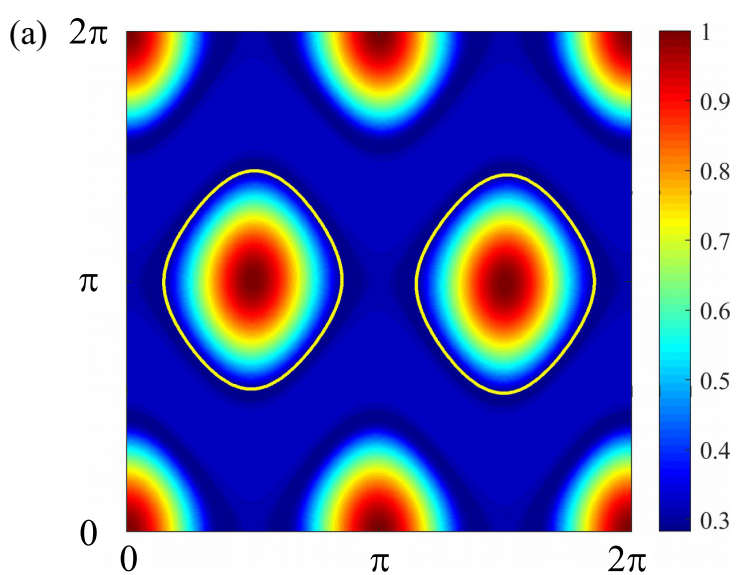

(b)

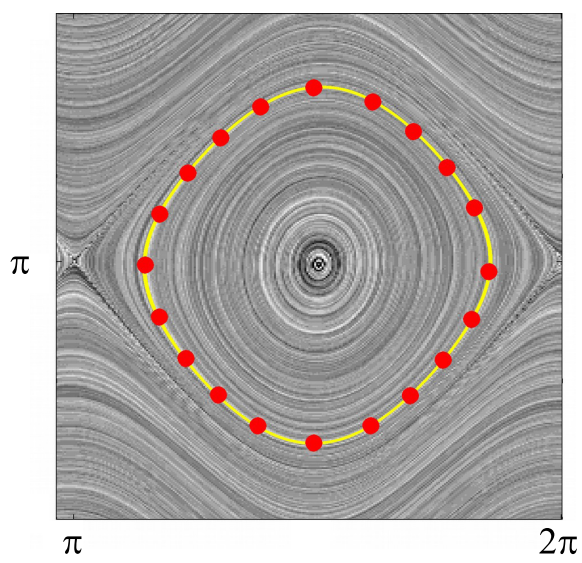

FIG. 6. Orszag-Tang IACD at $t_{0}$ with $\xi=100$ : (a) the normalized IACD field and the vortices detected as convex closed curves from the contour plot of IACD and (b) the line integral convolution plot of the magnetic field lines (dark gray) nearby one of the vortices. The circles are initial conditions on the vortex detected by IACD and the yellow lines are the corresponding magnetic field lines obtained by solving Eq. (2).

where the relation $\mathbf{Q}^{\prime} \mathbf{Q}^{\top}=-\mathbf{Q Q}^{\prime}{ }^{\top}$ was used [21]. The current density tensor $\mathbf{J}$, being proportional to tensor $\mathbf{A}$, Eq. (18), is also not objective.

Although the antisymmetrical tensor $\mathbf{A}$ is not objective, the averaged deviation is an objective quantity:

$$
\begin{aligned}
\overline{\mathbf{A}}-\langle\overline{\mathbf{A}}\rangle & =\mathbf{Q} \mathbf{A} \mathbf{Q}^{\top}+\mathbf{Q}^{\prime} \mathbf{Q}^{\top}-\left\langle\mathbf{Q} \mathbf{A} \mathbf{Q}^{\top}+\mathbf{Q}^{\prime} \mathbf{Q}^{\top}\right\rangle \\
& =\mathbf{Q} \mathbf{A} \mathbf{Q}^{\top}+\mathbf{Q}^{\prime} \mathbf{Q}^{\top}-\mathbf{Q}\langle\mathbf{A}\rangle \mathbf{Q}^{\top}-\mathbf{Q}^{\prime} \mathbf{Q}^{\top} \\
& =\mathbf{Q}(\mathbf{A}-\langle\mathbf{A}\rangle) \mathbf{Q}^{\top} .
\end{aligned}
$$

Although we have shown that the averaged deviation of the antisymmetrical tensor is objective, we are interested in the objectivity of the current density tensor. For this purpose, let us write the tensor $\mathbf{A}$ in Eq. (18) in index notation,

$$
\begin{aligned}
A_{i j} & =-\frac{\mu_{0}}{2} \varepsilon_{i j k} J_{k}, \\
A_{i j} e_{j} & =-\frac{\mu_{0}}{2} \varepsilon_{i j k} J_{k} e_{j}=\frac{\mu_{0}}{2} \varepsilon_{i k j} J_{k} e_{j}, \\
\mathbf{A} \boldsymbol{e} & =\frac{\mu_{0}}{2}(\boldsymbol{J} \times \boldsymbol{e}),
\end{aligned}
$$




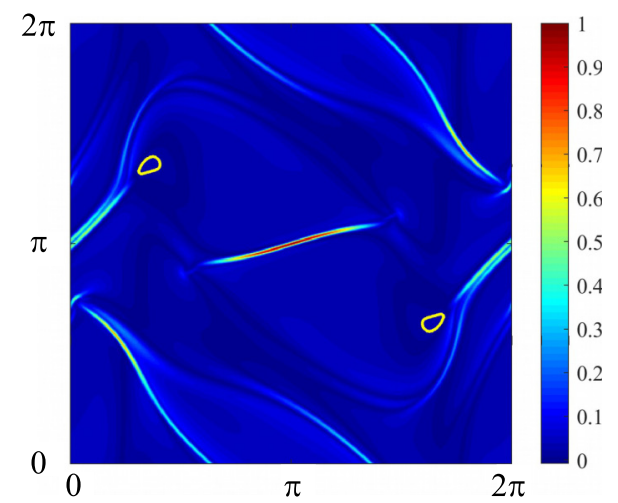

FIG. 7. Orszag-Tang current density at $t=1$ : the normalized magnitude of the current density and the small vortices detected as convex closed curves (rings) from the contour plot of $|\boldsymbol{J}|$.

where $\boldsymbol{e}$ is an arbitrary objective vector in $\mathbb{R}^{3}, \varepsilon_{i j k}$ is the Levi-Civita tensor [22], and $\boldsymbol{J}$ is the current density vector associated to tensor $\mathbf{A}$.

From Eq. (24),

$$
\mathbf{A} \boldsymbol{e}=\mathbf{Q}^{\top} \overline{\mathbf{A}} \mathbf{Q} \boldsymbol{e}-\mathbf{Q}^{\top} \mathbf{Q}^{\prime} \boldsymbol{e} .
$$

Now, using the objective transformation $\overline{\boldsymbol{e}}=\mathbf{Q} \boldsymbol{e}$ we can rewrite Eq. (27) as

$$
\mathbf{A} \boldsymbol{e}=\mathbf{Q}^{\top} \overline{\mathbf{A}} \overline{\boldsymbol{e}}-\mathbf{Q}^{\top} \mathbf{Q}^{\prime} \mathbf{Q}^{\top} \overline{\boldsymbol{e}}
$$

Analogously, we can define a vector $\alpha$ associated with the tensor $\mathbf{Q}^{\prime} \mathbf{Q}^{\top}$. Following in Eq. (26), since $\mathbf{Q}^{\prime} \mathbf{Q}^{\top}$ is an antisymmetrical tensor, its dual vector $\alpha$ is a non-null vector uniquely defined by [23]

$$
\mathbf{Q}^{\prime} \mathbf{Q}^{\top} \overline{\boldsymbol{e}}=\frac{\mu_{0}}{2}(\alpha \times \overline{\boldsymbol{e}}) .
$$

By substituting Eqs. (26) and (29) into Eq. (28) we obtain

$$
\begin{aligned}
\frac{\mu_{0}}{2}(\boldsymbol{J} \times \boldsymbol{e}) & =\mathbf{Q}^{\top} \frac{\mu_{0}}{2}(\overline{\boldsymbol{J}} \times \overline{\boldsymbol{e}})-\left[\mathbf{Q}^{\top} \frac{\mu_{0}}{2}(\alpha \times \overline{\boldsymbol{e}})\right] \\
\boldsymbol{J} \times \boldsymbol{e} & =\mathbf{Q}^{\top}(\overline{\boldsymbol{J}} \times \overline{\boldsymbol{e}})-\mathbf{Q}^{\top}(\alpha \times \overline{\boldsymbol{e}}) \\
\boldsymbol{J} \times \boldsymbol{e} & =\left[\left(\mathbf{Q}^{\top} \overline{\boldsymbol{J}}\right) \times\left(\mathbf{Q}^{\top} \overline{\boldsymbol{e}}\right)\right]-\left[\left(\mathbf{Q}^{\top} \alpha\right) \times\left(\mathbf{Q}^{\top} \overline{\boldsymbol{e}}\right)\right] \\
\boldsymbol{J} \times \boldsymbol{e} & =\left(\mathbf{Q}^{\top} \overline{\boldsymbol{J}}\right) \times \boldsymbol{e}-\left(\mathbf{Q}^{\top} \alpha\right) \times \boldsymbol{e} \\
\boldsymbol{J} \times \boldsymbol{e} & =\left[\mathbf{Q}^{\top} \overline{\boldsymbol{J}}-\mathbf{Q}^{\top} \alpha\right] \times \boldsymbol{e} \\
\boldsymbol{J} & =\mathbf{Q}^{\top} \overline{\boldsymbol{J}}-\mathbf{Q}^{\top} \alpha .
\end{aligned}
$$

The transformation described in Eq. (30) demonstrates that the current density vector is not an objective quantity. However, using Eq. (30), we obtain that the averaged current deviation is an objective vector:

$$
\begin{aligned}
\boldsymbol{J}-\langle\boldsymbol{J}\rangle & =\mathbf{Q}^{\top} \overline{\boldsymbol{J}}-\mathbf{Q}^{\top} \alpha-\left\langle\mathbf{Q}^{\top} \overline{\boldsymbol{J}}-\mathbf{Q}^{\top} \alpha\right\rangle \\
& =\mathbf{Q}^{\top} \overline{\boldsymbol{J}}-\mathbf{Q}^{\top} \alpha-\mathbf{Q}^{\top}\langle\overline{\boldsymbol{J}}\rangle+\mathbf{Q}^{\top} \alpha \\
& =\mathbf{Q}^{\top}(\overline{\boldsymbol{J}}-\langle\overline{\boldsymbol{J}}\rangle) .
\end{aligned}
$$

Therefore, the IACD method, Eq. (11), is objective, for

$$
|\overline{\boldsymbol{J}}-\langle\overline{\boldsymbol{J}}\rangle|=|\mathbf{Q}(\boldsymbol{J}-\langle\boldsymbol{J}\rangle)|=|\boldsymbol{J}-\langle\boldsymbol{J}\rangle| .
$$

(a) $2 \pi$

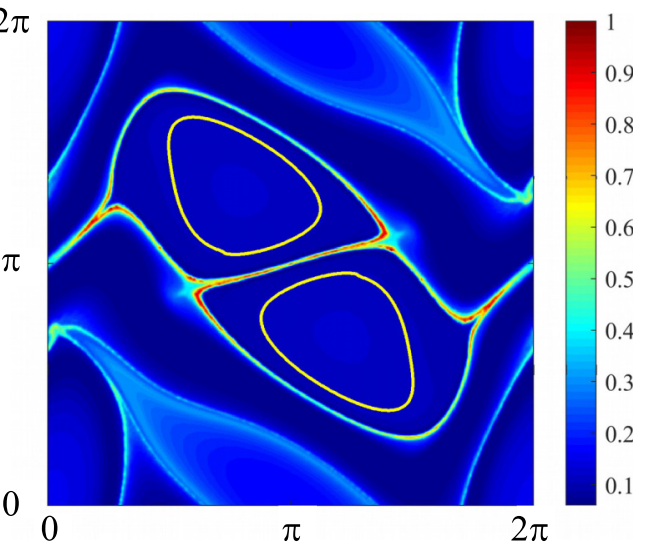

(b) $2 \pi$

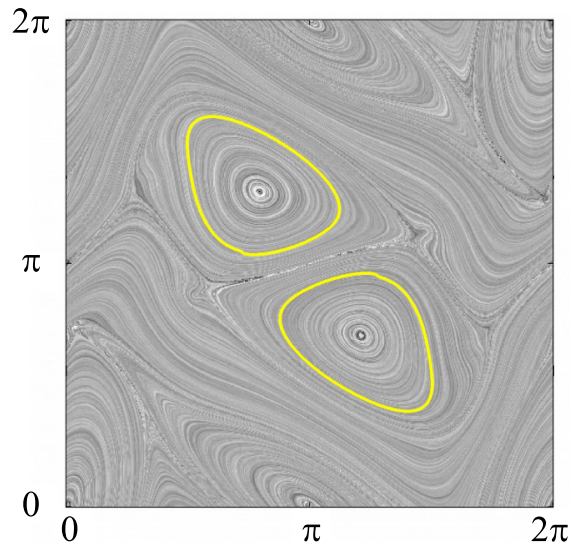

FIG. 8. Orszag-Tang IACD at $t=1$, with $\xi=25$ : (a) the normalized IACD field and the vortices detected as convex closed curves from the contour plot of IACD and (b) the line integral convolution plot of the magnetic field lines (dark gray) and the field lines starting from initial conditions (light gray [yellow online] circles) on an IACD vortex boundary shown in (a).

Note that the mean value $\langle\boldsymbol{J}\rangle$ is also not an objective quantity, since by taking averages in Eq. (30) we have

$$
\langle\boldsymbol{J}\rangle=\mathbf{Q}^{\top}\langle\overline{\boldsymbol{J}}\rangle-\mathbf{Q}^{\top} \alpha .
$$

In the particular case when $\langle\boldsymbol{J}\rangle=0,\langle\overline{\boldsymbol{J}}\rangle=\alpha$, which is not null, since $\alpha$ is uniquely defined by Eq. (29), where $\mathbf{Q}$ is an arbitrary rotation matrix.

\section{RESULTS}

\section{A. Two-dimensional incompressible MHD}

In dimensionless conservative form, the ideal MHD equations for an incompressible fluid are

$$
\begin{aligned}
& \frac{\partial \boldsymbol{u}}{\partial t}=-\nabla p-\nabla \cdot(\boldsymbol{u} \boldsymbol{u}-\boldsymbol{B B}), \\
& \frac{\partial \boldsymbol{B}}{\partial t}=-\nabla \cdot(\boldsymbol{u B}-\boldsymbol{B} \boldsymbol{u}), \\
& \nabla \cdot \boldsymbol{u}=0 \\
& \nabla \cdot \boldsymbol{B}=0
\end{aligned}
$$

where $\boldsymbol{u}$ is the velocity, $\boldsymbol{B}$ is the magnetic field, and $p$ is the total pressure. Equations (34)-(37) are solved in a square 
(a) $2 \pi$

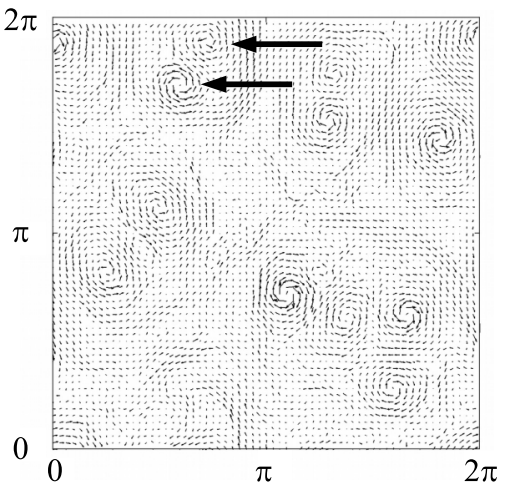

(b) $2 \pi$

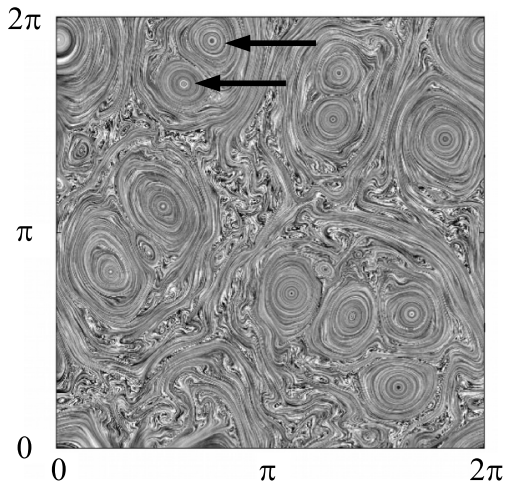

(d)

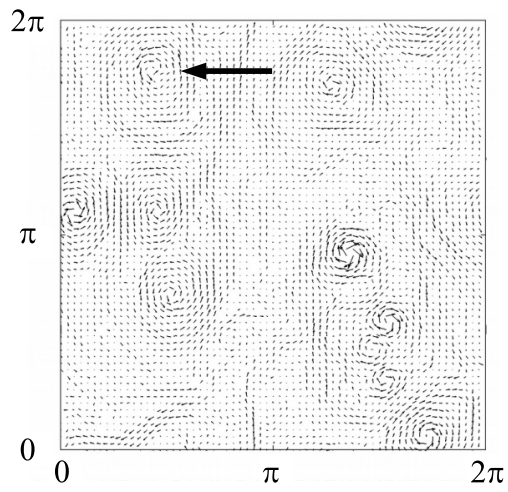

(e)

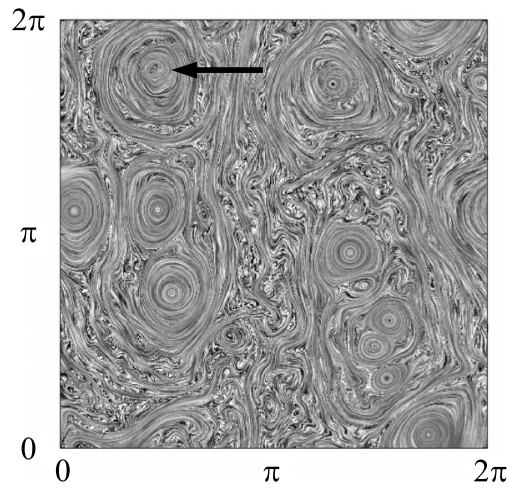

(c)

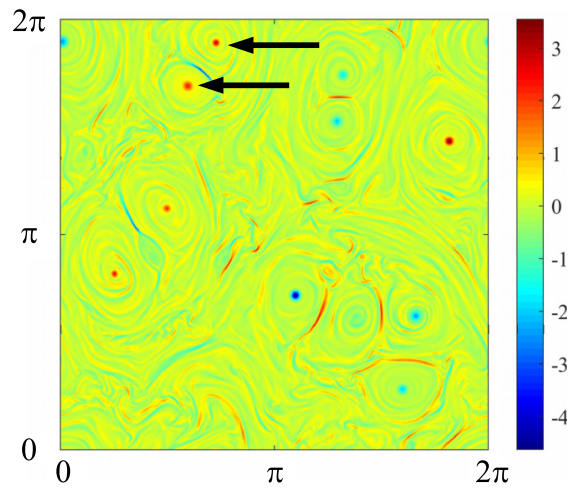

(f)

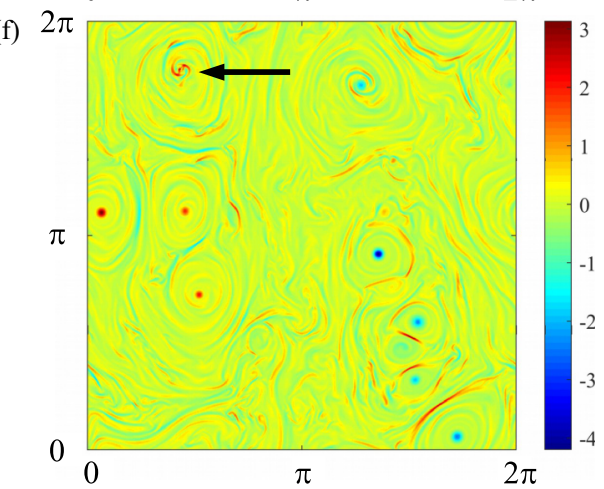

FIG. 9. Random vortices Eulerian fields at $t=300$ (upper panel) and $t=386$ (lower panel): (a, d) magnetic vectors, (b, e) magnetic field streamlines, and (c, f) current density.

domain with sides $L=2 \pi$ and periodic boundary conditions in both directions. Numerical integration is performed with a finite difference method proposed by Wu [24], where a fifthorder weighted essentially non-oscillatory (WENO) scheme is used in the spatial discretization, a variable-step fourthorder Runge-Kutta method is employed for time integration, and the incompressibility condition is enforced through the fractional-step method of Kim and Moin [25]. The numerical resolution is $512 \times 512$ grid points. In the following sections, Eqs. (34)-(37) are solved with two different sets of initial conditions.

\section{Deterministic initial conditions: Orszag-Tang vortex}

The Orszag-Tang (OT) vortex is defined by the following stream function $\psi$ and magnetic potential $a$ :

$$
\begin{gathered}
\psi(x, y)=2(\cos x+\cos y), \\
a(x, y)=\cos 2 x+2 \cos y .
\end{gathered}
$$

Figure 3 shows the state of the magnetic field at $t_{0}=0.01$, where Fig. 3(a) depicts the magnetic field vectors and Fig. 3(b) is an enlargement showing the $X$ point formed at the vortex interface. The magnetic field lines are exhibited in Fig. 3(c) as a line integral convolution (LIC) plot, which displays the integral curves of $\left(B_{x}, B_{y}\right)$ in different shades of gray. After one time unit, the magnetic field structures are distorted as in Fig. 4. The vectors near the interface between the two central vortices have become larger, thus increasing the magnetic shear and consequently enhancing the current density in that region.

As indicated by Eq. (11), the IACD is a measure of the deviation of the current density from its mean value, integrated along magnetic field lines. In order to distinguish the qualities of IACD from the current density itself, we compare the vortex detection using $\boldsymbol{J}$ and IACD. Figure 5 illustrates the detection using $\boldsymbol{J}$ at $t_{0}$. Figure 5(a) shows a color plot of $|\boldsymbol{J}|$ (normalized by $\max (|\boldsymbol{J}|)$ ) with two vortices indicated by black lines. Those vortices were found by employing the algorithm described in Sec. II B, where first a local maximum of the magnitude of the current density field is found, then the outermost closed convex contour line surrounding that maximum is extracted using MATLAB's contourc function. Figure 5(b) shows the magnetic field streamlines superposed by one of the two vortices (red line). The yellow spots are initial conditions from where the magnetic field lines are computed using Eq. (2). It can be seen that most lines spread away from the vortex region; therefore, the coherent structure identified from $\boldsymbol{J}$ is not a good representation of a magnetic vortex.

Next, we compute the IACD field at $t_{0}$ using Eq. (11) with $\xi=100$ and a convexity deficiency of $\varepsilon=10^{-3}$; the result is shown in Fig. 6. The top panel is the IACD field (normalized by $\max (\mathrm{IACD})$ ) and the vortices centered on two local maxima, denoted by the yellow lines. When initial conditions for Eq. (2) are chosen on the outermost convex contour surrounding an IACD maximum (red spots in the lower panel), the resulting magnetic field lines (yellow) remain confined to the vortical area, just inside the region surrounded by 
(a)

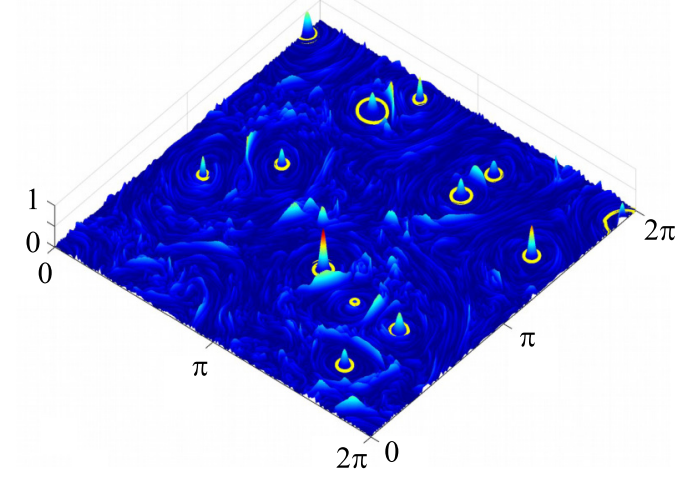

(b)

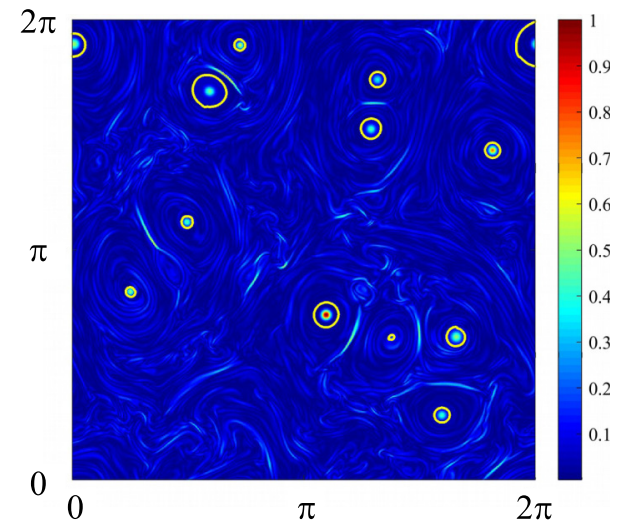

FIG. 10. Random vortices current density field at $t=300$ : (a) surface plot of the $|\mathbf{J}|$ field with rings denoting the outermost convex closed level curves surrounding selected local maxima; (b) the same as (a) but in a 2D color plot.

the separatrix lines starting at the two neighboring $X$ points, which can be seen in the LIC plot.

When the current density and IACD are applied to vortex detection at $t=1$, the results are as shown in Figs. 7 and 8, respectively. The current density failed to identify large vortices in the domain, as the main coherent structures are the current sheets formed at the vortex interfaces due to the strong magnetic shear. The shear also impacts the vortex detection using IACD, since its computation is based on $|\boldsymbol{J}|$. Nonetheless, at $t=1$ it still manages to extract the main vortical regions, as seen in Fig. 8. For larger $t$, the shear increases even further, which impacts the numerical computation of the closed contours; thus thresholds in $|\boldsymbol{J}|$ need to be imposed if one wants to use these techniques to find the vortical structures. Since IACD is based on the curl of $\boldsymbol{B}$, it will be large on regions with rotation of magnetic field lines as well as shear regions, a problem shared with the LAVD operator in velocity fields. For a discussion on problems related to kinematic vortex detection in strong shear regions of observational velocity fields using LAVD, as well as possible solutions to these problems, see Silva et al. [26].

We now proceed with a different example where the initial conditions produce a result that may be more relevant to turbulence studies. (a)

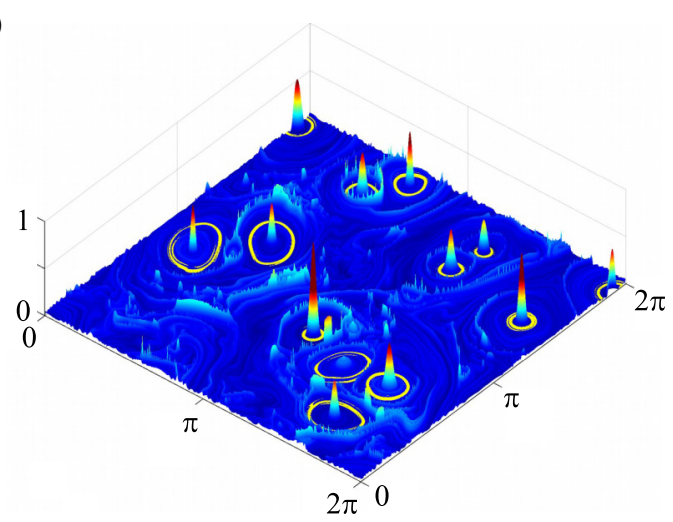

(b)

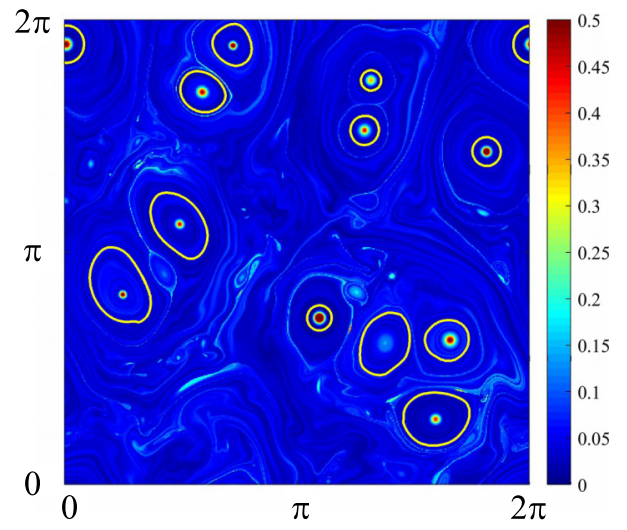

FIG. 11. Random vortices IACD at $t=300$ from $s_{0}=0$ to $s_{0}+\xi$, with $\xi=100$ : (a) surface plot of the IACD field with rings denoting the outermost convex closed level curves surrounding selected local maxima; (b) the same as (a) but in a 2D color plot.

\section{Random initial conditions: Wu-Chang model of magnetotail}

In this section, the MHD model for vortex interactions presented by $\mathrm{Wu}$ and Chang [7] is adopted to test the IACD method. The initial conditions are given by random velocity and magnetic fields, where the magnetic field is specified by a flux function. In the Fourier space, the two components of the velocity and the magnetic flux function are proportional to $k^{2} \exp \left(-\alpha k^{2}\right)$, where $\alpha=0.01$ is adopted, so the initial kinetic energy spectrum is peaked at $k=10$. The initial condition is such that the maximum amplitude is 0.2 for the velocity field and 0.3 for the magnetic field.

Figure 9 depicts the system state at $t=300$ (upper panels) and $t=386$ (lower panels). Figures 9(a) and 9(d) plot the magnetic field vectors, Figs. 9(b) and 9(e) are line integral convolution (LIC) plots, showing the instantaneous streamlines of the magnetic field, and Figs. 9(c) and 9(f) show the current density plots. The arrows in the upper panel point to a couple of magnetic vortices that move with the flow until they coalesce and merge into a single vortex, indicated by arrows in the lower panel.

In order to show this vortex-vortex interaction in more detail, we proceed to extract the vortex boundaries by applying the algorithm described in Sec. II B. First, we perform the analysis using the current density alone. Thus, a local maximum of the magnitude of the current density field is found 


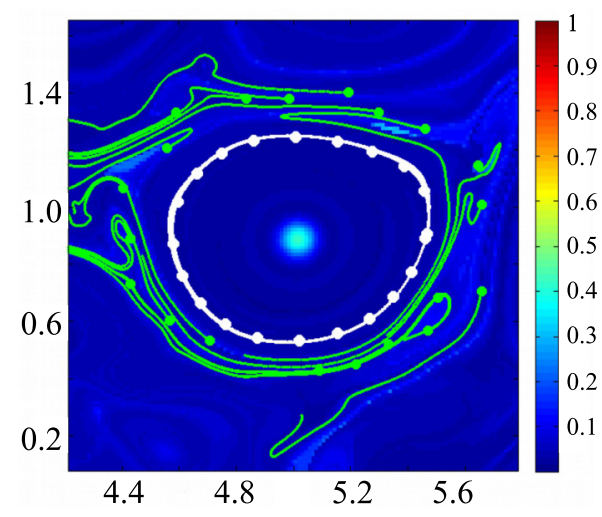

FIG. 12. Random vortices magnetic field lines at $t=300$ from $s_{0}=0$ to $s_{0}+\xi$, with $\xi=100$. The white lines denote the field lines starting from initial conditions (white circles) on an IACD vortex; the light gray (green online) lines denote field lines starting from surrounding initial conditions.

and the outermost closed convex contour line surrounding that maximum is extracted. Figure 10 shows two views of the magnitude of the current density at $t=300$ with a set of vortices found with the given algorithm, where a convexity deficiency $\varepsilon=10^{-3}$ was adopted. A comparison between Figs. 9(b) and 10(b) seems to suggest that the numerically computed vortex boundaries from the $|\boldsymbol{J}|$ field are underestimated and wider vortical regions should be expected. The choice of larger values of $\varepsilon$ (within reasonable limits) does not significantly change the results, which are related to the intrinsic ruggedness of the $|\boldsymbol{J}|$ field. This problem is not present in the IACD vortices, shown in Fig. 11, where the IACD field is computed at time $t=300$ from Eq. (11) with $s_{0}=0$ and $\xi=100$. The IACD method managed to correctly detect the magnetic vortex boundaries, outside which the magnetic field lines will tend to disperse away. This can be seen in Fig. 12, which shows an enlargement of the rectangular region in Fig. 11. The white circles denote a set of initial conditions on the vortex boundary computed from the IACD field; the white lines represent the trajectories of these initial conditions under the magnetic field line equation [Eq. (2)]. Note that all magnetic field lines stay together in a coherent bundle surrounding the vortex center for $\xi=100 \mathrm{~s}$ units. However, when we start from initial conditions outside the IACD vortex (green circles), the resulting magnetic field lines spread away in different directions (green lines). Therefore, the IACD method can detect magnetic vortex boundaries more precisely than the $|\boldsymbol{J}|$ method in this example.

Back to the previously mentioned vortex merging, Fig. 13 shows an enlargement of the upper left corner of the domain box, where the merging pointed out in Fig. 9 occurs. The upper panels represent the magnetic vectors and the lower panels the LIC plots of the magnetic streamlines at different times, until the two vortices coalesce into a larger vortex. These coalescence events are important because they form local sites of enstrophy production, conversion, and dissipation [3]. The boundaries of these vortices were computed with IACD using $\xi=100$ and are shown in Fig. 14, in both color scales [Figs. 14(a)-14(d)] and surface plots [Figs. 14(e)-14(h)]. The moment when the merging is effected can be defined by the presence of only one peak in the IACD surface plot in Fig. 14(h).

\section{B. Three-dimensional compressible MHD}

The previous section showed that the IACD can be used to accurately detect magnetic vortex boundaries in planar fields, where the structures detected were shown to match the LIC (a)

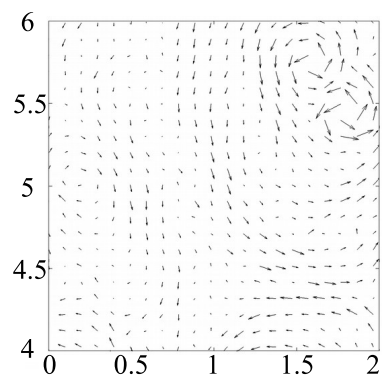

(e)

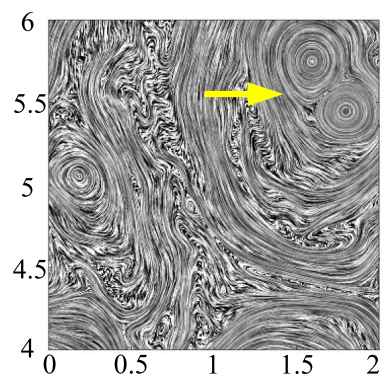

(b)

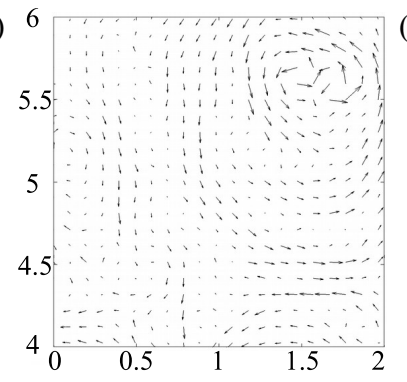

(f)

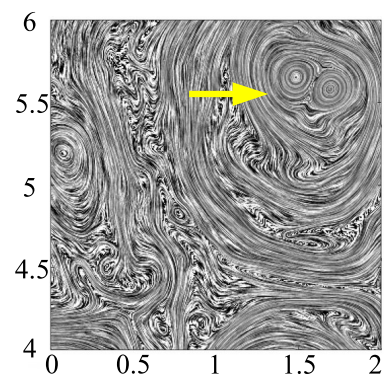

(c)

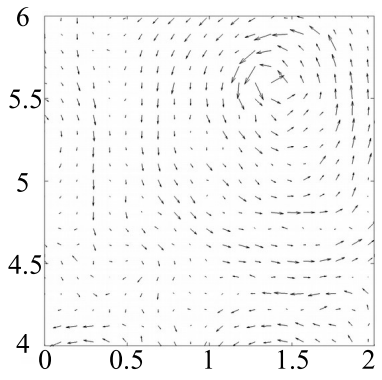

(g)

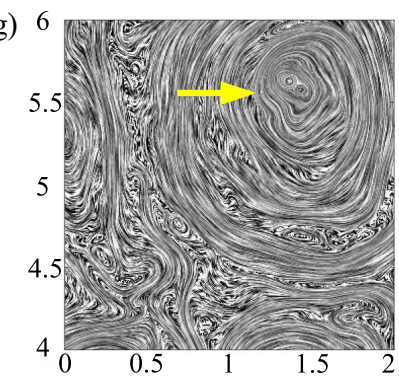

(d)

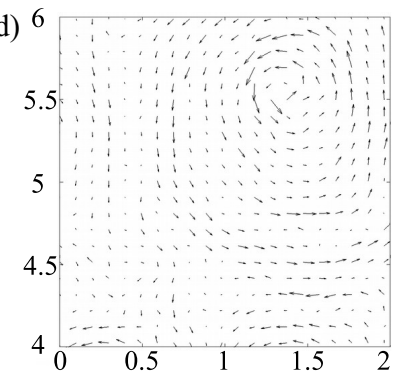

(h)

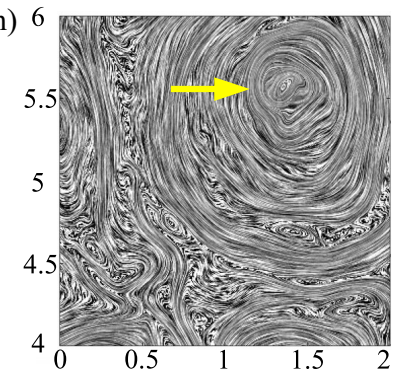

FIG. 13. Random magnetic vortices merging: magnetic field vectors at (a) $t=362.34$, (b) $t=372.32$, (c) $t=384.54$, and (d) $t=386.82$, respectively; $(\mathrm{e}-\mathrm{h})$ the line integral convolution plots corresponding to the magnetic field lines of $(\mathrm{a}-\mathrm{d})$. The arrow points to two vortices that coalesce at $t=386.82$. 
(a) 6

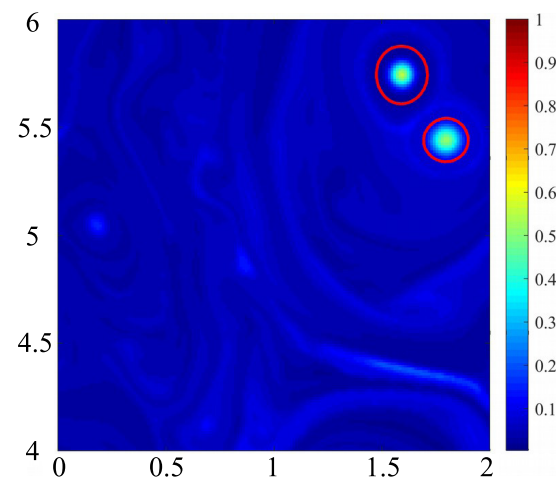

(c)

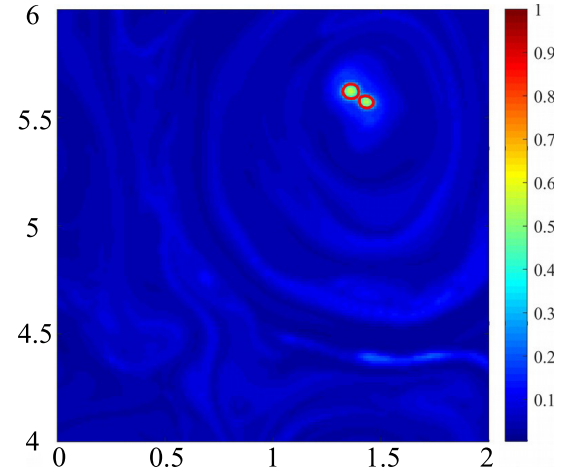

(e)

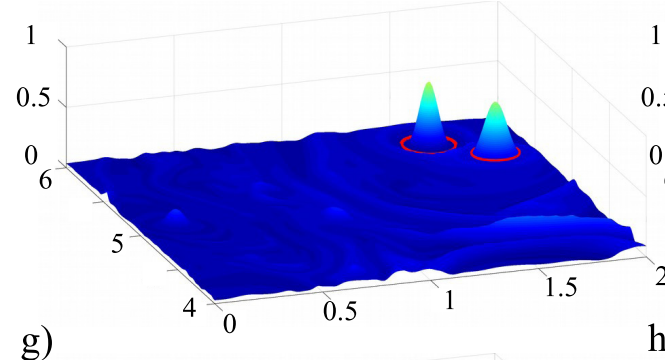

g)

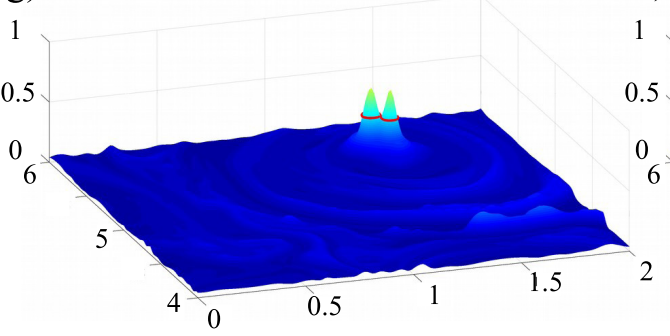

(b)

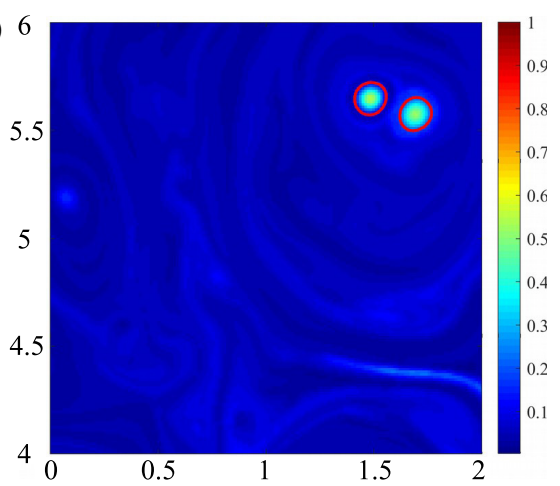

(d)

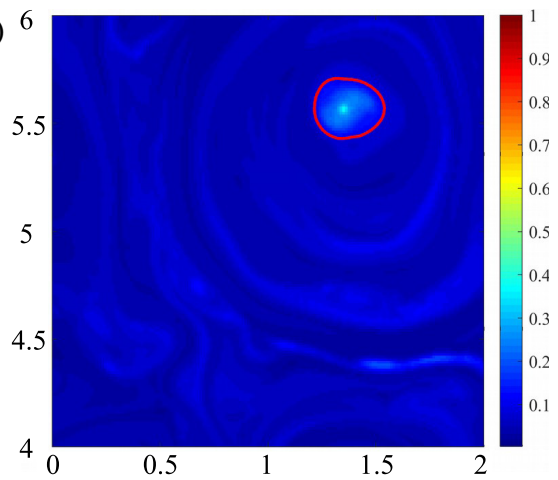

(f)

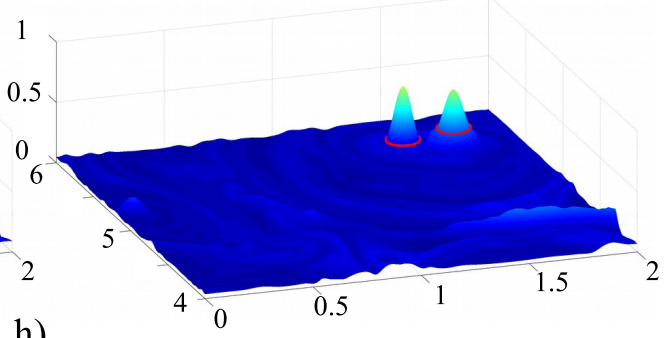

h)

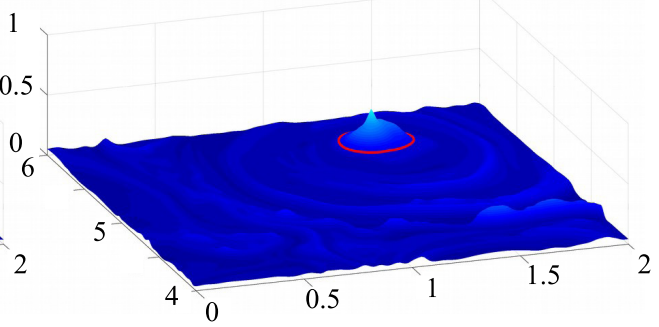

FIG. 14. Random magnetic vortices merging in the IACD field: magnetic vortices detected from the IACD field at (a) $t=362.34$, (b) $t=372.32$, (c) $t=384.54$, and (d) $t=386.82$, respectively; (e-h) surface plots of IACD corresponding to (a-d).

plots of the magnetic streamlines. However, the qualities of IACD can be better appreciated in $3 \mathrm{D}$ fields. In this section, the IACD operator is employed in detecting coherent magnetic structures in three-dimensional numerical simulations of the resistive MHD equations for a compressible isothermal gas. The model equations are the same as used in [27] for simulations of hydromagnetic turbulence,

$$
\begin{aligned}
& \frac{\partial \boldsymbol{u}}{\partial t}+\boldsymbol{u} \cdot \nabla \boldsymbol{u}=-\frac{\nabla p}{\rho}+\frac{\boldsymbol{J} \times \boldsymbol{B}}{\rho}+\boldsymbol{F}_{\mathrm{visc}} \\
& \frac{\partial \boldsymbol{A}}{\partial t}=\boldsymbol{u} \times \boldsymbol{B}+\eta \nabla^{2} \boldsymbol{A}
\end{aligned}
$$

$$
\frac{\partial \ln \rho}{\partial t}+\boldsymbol{u} \cdot \nabla \ln \rho=-\nabla \cdot \boldsymbol{u}
$$

where $\boldsymbol{u}$ is the velocity, $\boldsymbol{B}$ is the magnetic field, $\rho$ is the density, $c_{s}$ is the isothermal sound speed, $\boldsymbol{p}$ is the pressure, $\boldsymbol{J}=\nabla \times \boldsymbol{B} / \mu_{0}$ is the current density, with $\mu_{0}$ the constant vacuum permeability, $\boldsymbol{A}$ is the magnetic vector potential, and $\eta$ is the constant magnetic diffusivity. The pressure gradient is written as $\nabla p / \rho=c_{s}^{2} \nabla \ln \rho$, where $c_{s}^{2}=p / \rho$ is constant. The viscous term is given by $\boldsymbol{F}_{\text {visc }}=v\left(\nabla^{2} \boldsymbol{u}+\nabla \nabla \cdot \boldsymbol{u} / 3+\right.$ $2 \mathbf{S} \cdot \nabla \ln \rho$ ), where $v$ is the constant kinematic viscosity and $\mathbf{S}$ 


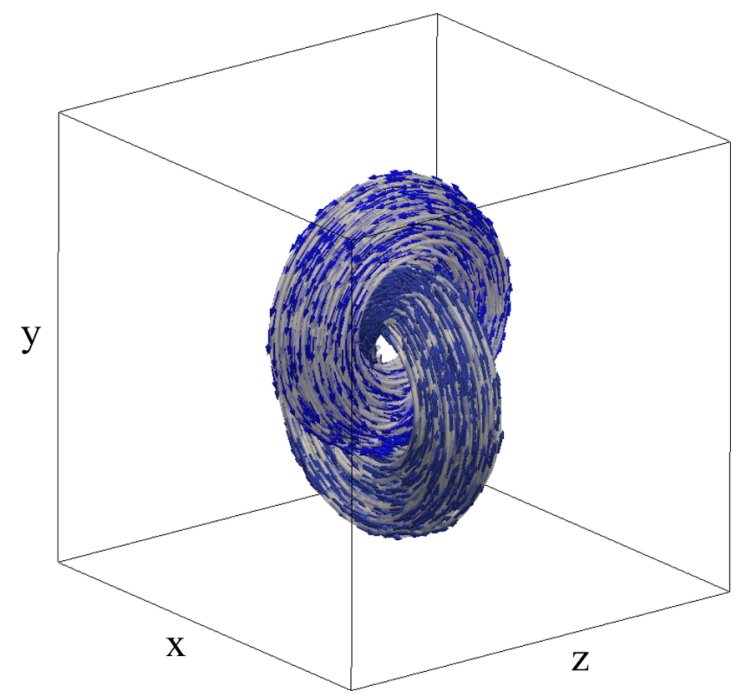

FIG. 15. Interlocked magnetic flux rings at $t=0$.

is the traceless rate of strain tensor,

$$
\mathrm{S}_{i j}=\frac{1}{2}\left(\frac{\partial u_{i}}{\partial x_{j}}+\frac{\partial u_{j}}{\partial x_{i}}-\frac{2}{3} \delta_{i j} \nabla \cdot \boldsymbol{u}\right)
$$

(a)

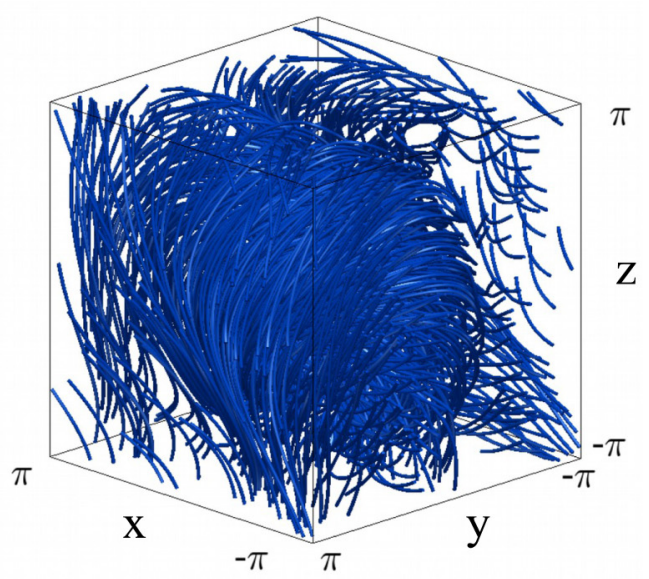

(b)

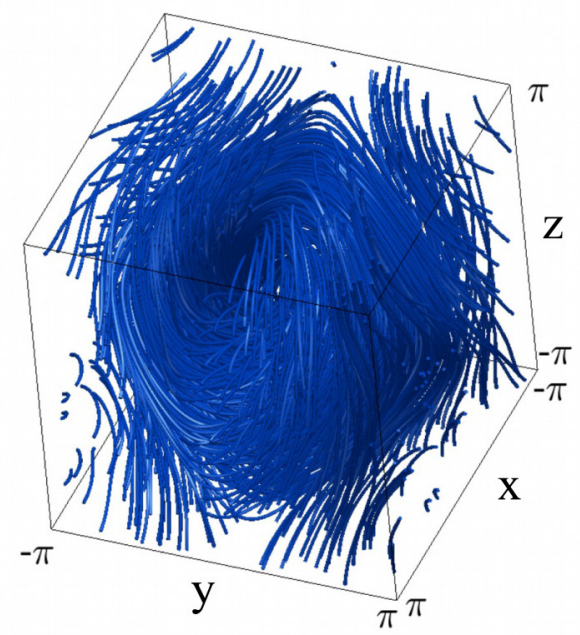

FIG. 16. Magnetic field lines from random initial conditions at $t=100$ : (a, b) two views of the same data from different angles.
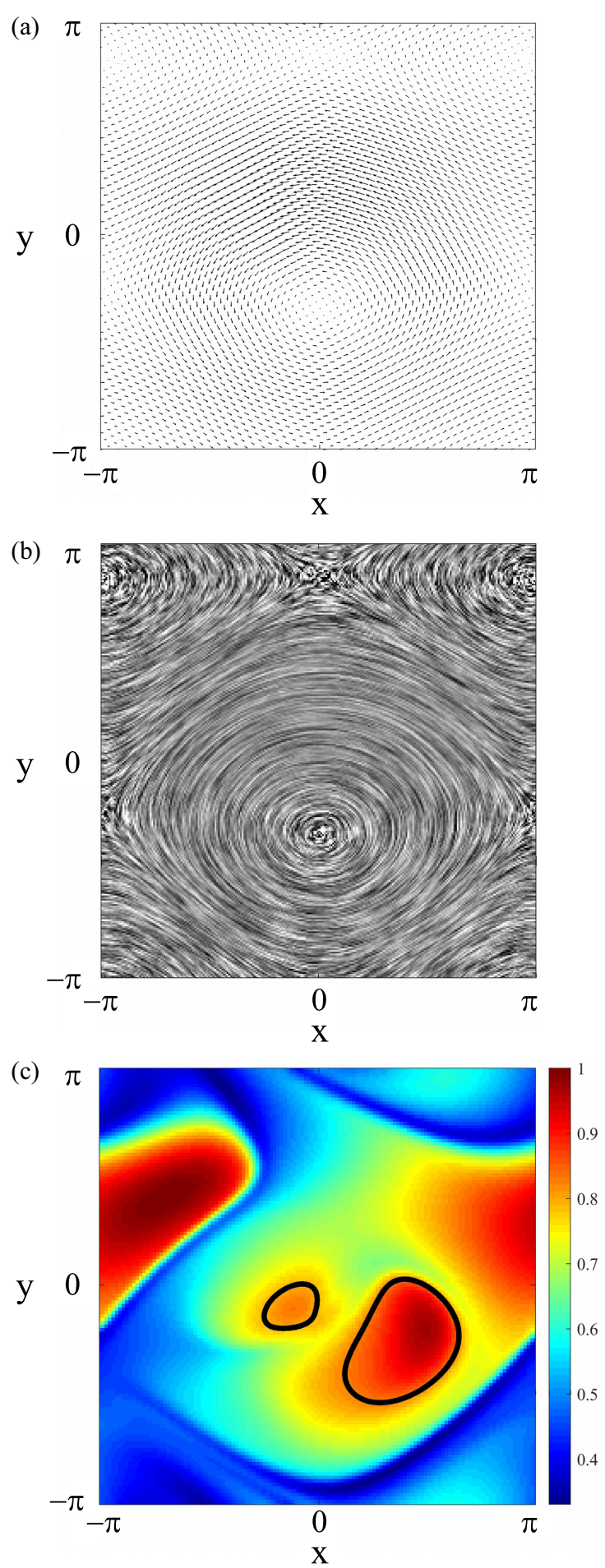

FIG. 17. Horizontal magnetic field components on $z=0$ at $t=100$ : (a) horizontal components $\left(B_{x}, B_{y}\right)$ of the magnetic field vectors, (b) two-dimensional streamlines of the magnetic field computed using only the horizontal components, and (c) the IACD field with two magnetic vortices (black lines).

where $\delta_{i j}$ is the Kronecker delta. The domain is a square box with sides $L=2 \pi$ and periodic boundary conditions, which implies that mass is conserved, $\langle\rho\rangle=\rho_{0}$, where $\rho_{0}$ is the initial uniform density and the brackets denote volume average. Nondimensional quantities are adopted where length 
is in units of $L / 2 \pi, \boldsymbol{u}$ is in units of $c_{S}, \rho$ is in units of $\rho_{0}$, and $\boldsymbol{B}$ is in units of $\left(\mu_{0} \rho_{0} c_{s}^{2}\right)^{1 / 2}$.

Equations (40)-(42) are solved with sixth-order centered finite differences in space and third-order variable-step Runge-Kutta in time, using the PENCIL CODE [28] in a square box with sides $L=2 \pi$ and periodic boundary conditions. The initial conditions are $\boldsymbol{u}=\mathbf{0}, \rho=\rho_{0}=1$. The magnetic initial condition consists of a pair of interlocked magnetic tori, or flux rings, both with the same flux and with inner radius $R_{i}=0.3$ and outer radius $R_{o}=1$, as seen in Fig. 15; inside the tori $\nabla \times \boldsymbol{B}=\mathbf{0}$ and the initial $\boldsymbol{B}$ profile across each ring is a Gaussian. One torus is aligned with the plane $z=0$ and the other with the plane $x=0$. The initial tori setup is provided with the PENCIL CODE and described in the code's manual; variations of this problem with two or three interlocked rings were previously studied by Kerr and Brandenburg [29] and Del Sordo et al. [30], respectively. We set $\eta=v=0.003$ and adopt a numerical resolution of $128^{3}$ grid points.

The tension due to the curvature of the flux tubes initially shrinks the rings and induces a velocity [29]. Eventually, magnetic reconnection takes place and the topology of the field lines is changed. The configuration of the magnetic field lines after $t=100$ time units is shown in Fig. 16. The complex entanglement plotted from random initial conditions hides the inherent order present in the magnetic coherent structures that are still embedded in the field. We start searching for those structures by considering their horizontal components on the plane which sections the middle of the box at $z=0$. Figures 17(a) and 17(b) show the horizontal components of the magnetic field vectors $\left(B_{x}, B_{y}\right)$ and their two-dimensional streamlines, respectively. The presence of two large swirling regions interacting via the periodic boundary conditions can be seen. However, this view of the field on the plane does not take into account the torsion of the lines in the $z$ direction, thus concealing the true complexity of the structures in the box. Figure $17(\mathrm{c})$ shows the $\mathrm{IACD}_{s_{0}=0}^{\xi=2000}\left(\boldsymbol{x}_{0}\right)$ scalar field, where $\boldsymbol{x}_{0}$ belongs to a grid of points on the plane and the closed black curves delineate two magnetic vortices located in the central region of the box. Note that, although the large swirling structure seen in Fig. 17(b) certainly has an impact on the coherence of the field lines in the short term, it is not the main convex coherent structure for a long integration of field lines with $\xi=2000$.

The coherence of the structures identified by IACD in Fig. 17(c) can be seen in Fig. 18, where we plot the magnetic field lines (red) emanating from the two vortex contours (black). The left panels refer to the smaller vortex of Fig. 17(c) and the right panels to the larger vortex. The lower panels are the same as the upper panels, but with the inclusion of a few magnetic field lines from random initial conditions. It is remarkable that IACD is able to detect the magnetic flux ropes among such a complex entanglement of field lines, especially considering that the boundary was calculated only on a plane.
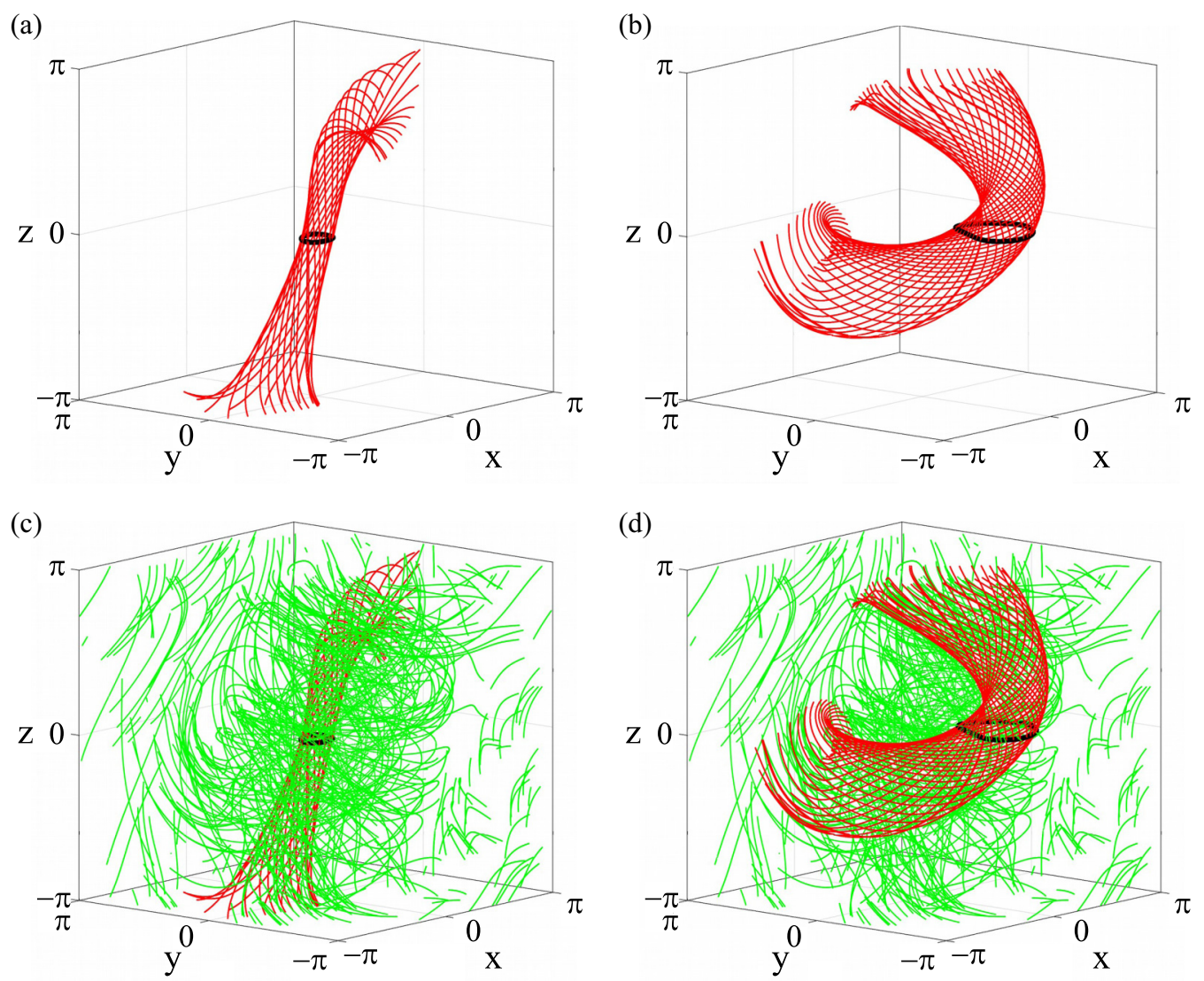

FIG. 18. Magnetic flux-rope reconstruction at $t=100$ with $\xi=2000$ : (a) magnetic field lines (dark gray [red online] lines) emanating from the small vortex boundary (black line), (b) magnetic field lines from the large vortex boundary; (c, d) the same as in (a) and (b), but with the inclusion of magnetic field lines emanating from random initial conditions (light gray [green online]). 
(a)

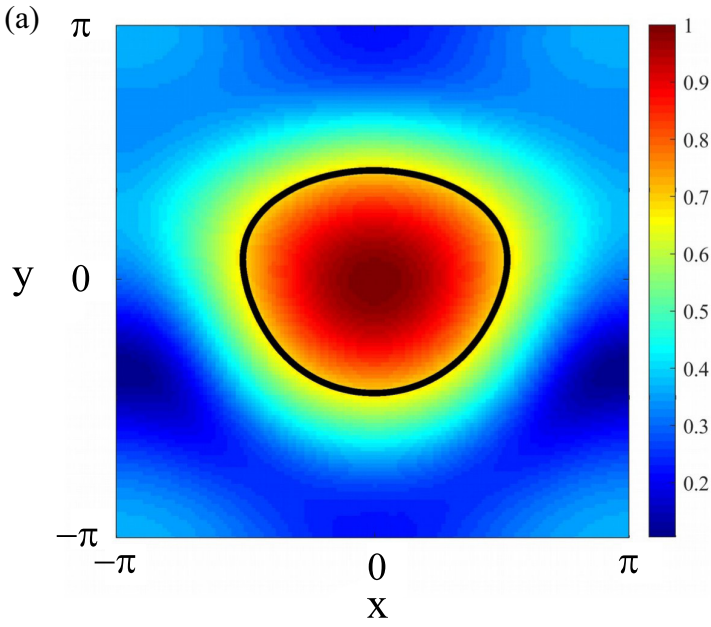

(b)

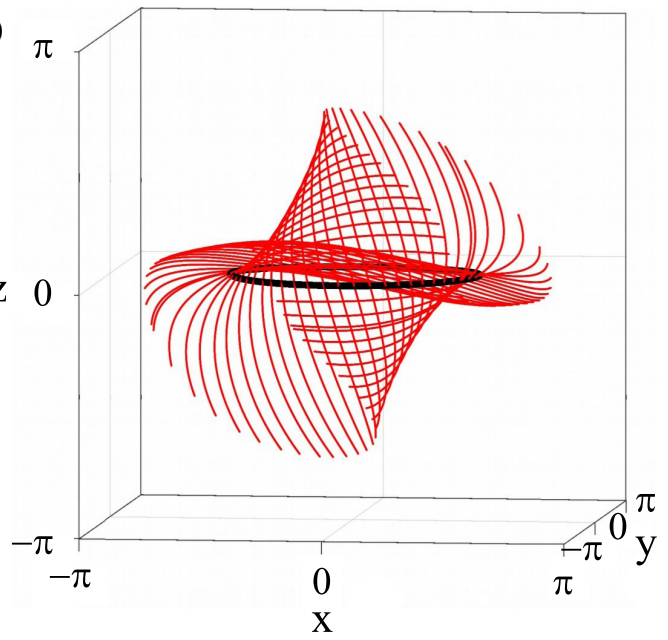

FIG. 19. Magnetic flux-rope reconstruction at $t=100$ with $\xi=500$ : (a) the IACD field on the midplane $z=0$, with one magnetic vortex (black line), and (b) magnetic field lines (red lines) emanating from the vortex boundary (black line).

As previously mentioned in Sec. II B 2, the parameter $\xi$ in Eq. (11) basically controls the length and shape of the vortices detected by IACD. In Fig. 19, we illustrate what happens when a smaller value of $\xi$ is adopted for the same magnetic field used in Figs. 17 and 18. The $\operatorname{IACD}_{s_{0}=0}^{\xi=500}\left(x_{0}\right)$ field in Fig. 19(a) is much simpler than in Fig. 17(a), since the field lines are short enough to stay away from the boundaries. A single wide vortex is found and a few magnetic field lines from its boundary are plotted in Fig. 19(b). The structure will lose coherence and the field lines will spread out if longer integration is conducted to compute the field lines.

\section{DISCUSSION AND CONCLUSIONS}

In the past decade, objective (observer-independent) Lagrangian techniques have prevailed in the detection of coherent structures in hydrodynamic flows by means of the theory of Lagrangian coherent structures (LCS), with a set of mathematical tools being developed to precisely define hyperbolic (manifold lines), parabolic (jet cores), and elliptic (vortices) LCS $[2,31]$. Following that tendency, a series of works has employed LCS techniques to detect structures in plasmas from numerical simulations of the MHD equations [32-34] as well as observational data of the solar photosphere $[26,35,36]$. In [14], the first definition of a magnetic vortex based on the theory of LCS was introduced in the form of the IACD operator, which was explored in the present paper by means of three MHD examples, namely, a two-dimensional incompressible Orszag-Tang vortex, two-dimensional incompressible random vortices, and three-dimensional compressible interlocked flux rings. The IACD was shown to precisely detect the outer contour of the coherent magnetic structures that keep the magnetic field lines bundled together, even among surrounding turbulence. A comparison with detection using the current density alone proved the superiority of the IACD, which is also invariant to spatially dependent rotations and translations of the observer, which the current density is not (see Sec. III). The method can be used in conjunction with the squashing factor Q [35-37] to investigate magnetic reconnection in simulated and observational plasmas, since the IACD can identify the main structures involved in the reconnection while the $\mathrm{Q}$ operator detects the quasi-separatrix layers, which are the main magnetic reconnection sites between those structures [12]. A possible application is the reconnection of the solar emerging flux ropes with the coronal field [38]. Detection of the boundary layers in interacting magnetic flux ropes is also crucial for understanding the origin of intermittent turbulence in the solar wind, with resulting non-Gaussian statistics and cross-scale coupling $[39,40]$. Additionally, flux ropes are found in coronal mass ejections (CMEs), giant releases of plasma from the Sun. These flux ropes are formed in the low corona and carried away through the interplanetary medium, where they can reconnect with magnetospheres [41].

The vortex merging observed in our two-dimensional random vortex simulations (Sec. IV A 2) was previously applied by Wu and Chang [7] to the observation of bursty bulk flows in the Earth's magnetotail. In fact, sequential releases of tilted flux ropes due to reconnection were observed in the Earth's magnetotail by Hietala et al. [42], where the magnetic flux ropes were reconstructed by a combination of two-spacecraft timing for their global orientation and Grad-Shafranov reconstruction (GSR) for their local orientation. The GSR method models the magnetic field structure traversed by a spacecraft [43] and is the same technique employed in [39] to reconstruct interplanetary magnetic flux ropes. Another example of the use of the GSR method is the reconstruction of flux ropes from ACE and Wind spacecraft data, which was recently used to study flux ropes merging in the solar wind by Zheng et al. [44]. This renders support for the use of IACD not only in numerical simulations, but also in observational data. Perhaps an even better natural laboratory for this technique is the Sun, where the vector magnetic field can be measured by space instruments such as the Helioseismic and Magnetic Imager (HMI) onboard the Solar Dynamics Observatory (SDO) [45]; alternatively the horizontal magnetic field in the solar surface can be estimated from the photospheric flow, as proposed by Yeates et al. [35]. In conclusion, we believe that IACD is a novel and simple objective tool that can be used to detect magnetic vortices in numerical simulations and observations of space and astrophysical plasmas. 


\section{ACKNOWLEDGMENTS}

We are indebted to Dr. C. C. Wu of UCLA for kindly providing us his numerical codes for our 2D MHD simulations, without which this paper would have not been possible. We thank Dr. F. J. Beron-Vera for helpful discussions. This study was financed in part by the Coordenação de Aperfeiçoamento de Pessoal de Nível Superior - Brasil (CAPES), Finance Code
001. E.L.R. acknowledges Brazilian agencies CAPES, CNPq, and FAPESP (Grants No. 2013/26258-4 and No. 16/24970-7) for their financial support. S.S.A.S. and T.F.P.G. acknowledge Brazilian agency CAPES for the financial support. Research was carried out using the computational resources of the Center for Mathematical Sciences Applied to Industry (CeMEAI), funded by FAPESP (Grant No. 2013/07375-0).
[1] U. Frisch, Turbulence: The Legacy of A. N. Kolmogorov (Cambridge University Press, Cambridge, UK, 1995).

[2] G. Haller, A. Hadjighasem, M. Farazmand, and F. Huhn, J. Fluid Mech. 795, 136 (2016).

[3] R. Kinney, J. C. McWilliams, and T. Tajima, Phys. Plasmas 2, 3623 (1995).

[4] S. A. Orszag and C.-M. Tang, J. Fluid Mech. 90, 129 (1979).

[5] D. Biskamp and H. Welter, Phys. Fluids B 1, 1964 (1989).

[6] H. Politano, A. Pouquet, and P. L. Sulem, Phys. Fluids B 1, 2330 (1989).

[7] C.-C. Wu and T. Chang, J. Atmos. Terr. Phys. 63, 1447 (2001).

[8] C. K. Armstrong and I. J. D. Craig, Solar Phys. 283, 463 (2013).

[9] C. K. Armstrong and I. J. D. Craig, Solar Phys. 289, 869 (2014).

[10] V. S. Lukin, Plasma Phys. Controlled Fusion 56, 060301 (2014).

[11] J. Sears et al., Plasma Phys. Controlled Fusion 56, 095022 (2014).

[12] J. B. O'Bryan and C. R. Sovinec, Plasma Phys. Controlled Fusion 56, 064005 (2014).

[13] C. Lawder and A. Yeates, Astrophys. J. 846, 106 (2017).

[14] E. L. Rempel et al., Mon. Not. R. Astron. Soc. 466, L108 (2017).

[15] F. J. Beron-Vera, A. Hadjighasem, Q. Xia, M. J. Olascoaga, and G. Haller, Proc. Natl. Acad. Sci. USA (2018), doi:10.1073/pnas.1701392115.

[16] C. Prior and A. R. Yeates, Astrophys. J. 787, 100 (2014).

[17] C. Truesdell and W. Noll, The Nonlinear Field Theories of Mechanics (Springer, Berlin, 2004).

[18] T. Günther and H. Theisel, Comput. Graph. Forum 37, 149 (2018).

[19] G. Haller, J. Fluid Mech. 525, 1 (2005).

[20] S. Kotsiaros and N. Olsen, Int. J. Geomath. 3, 297 (2012).

$[21] \mathbf{Q} \mathbf{Q}^{\top}=\mathbf{Q Q}^{-1}=1 \Rightarrow\left(\mathbf{Q} \mathbf{Q}^{\top}\right)^{\prime}=\mathbf{Q}^{\prime} \mathbf{Q}^{\top}+\mathbf{Q} \mathbf{Q}^{\prime \top}=0 \Rightarrow \mathbf{Q}^{\prime} \mathbf{Q}^{\top}=$ $-\mathbf{Q Q}^{\prime \top}$.

[22] $\varepsilon_{123}=\varepsilon_{312}=\varepsilon_{231}=1, \varepsilon_{213}=\varepsilon_{312}=\varepsilon_{321}=-1$, and $\varepsilon_{i j k}=0$ for repeated indices.
[23] C. Fang, An Introduction to Fluid Mechanics (Springer International Publishing, Cham, 2019).

[24] C.-C. Wu, Geophys. Astrophys. Fluid Dyn. 101, 37 (2007).

[25] J. Kim and P. Moin, J. Comput. Phys. 59, 308 (1985).

[26] S. S. A. Silva et al., Astrophys. J. 863, L2 (2018).

[27] N. E. L. Haugen, A. Brandenburg, and W. Dobler, Phys. Rev. E 70, 016308 (2004).

[28] http://pencil-code.nordita.org/

[29] R. M. Kerr and A. Brandenburg, Phys. Rev. Lett. 83, 1155 (1999).

[30] F. DelSordo, S. Candelaresi, and A. Brandenburg, Phys. Rev. E 81, 036401 (2010).

[31] G. Haller, Annu. Rev. Fluid Mech. 47, 137 (2015).

[32] E. L. Rempel, A. C.-L. Chian, and A. Brandenburg, Astrophys. J. 735, L9 (2011).

[33] E. L. Rempel, A. C.-L. Chian, and A. Brandenburg, Phys. Scr. 86, 018405 (2012).

[34] E. L. Rempel et al., J. Fluid Mech. 729, 309 (2013).

[35] A. R. Yeates, G. Hornig, and B. T. Welsch, Astron. Astrophys. 539, A1 (2012).

[36] A. C.-L. Chian et al., Astrophys. J. 786, 51 (2014).

[37] V. S. Titov, G. Hornig, and P. Démoulin, J. Geophys. Res. 107, 1164 (2002).

[38] D. MacTaggart and A. L. Haynes, Mon. Not. R. Astron. Soc. 43856, 1500 (2014).

[39] A. C.-L. Chian et al., Astrophys. J. 832, 179 (2016).

[40] R. A. Miranda, A. B. Schelin, A. C.-L. Chian, and J. L. Ferreira, Ann. Geophys. 36, 497 (2018).

[41] A. Vourlidas, Plasma Phys. Controlled Fusion 56, 064001 (2014).

[42] H. Hietala, J. P. Eastwood, and A. Isavnin, Plasma Phys. Controlled Fusion 56, 064011 (2014).

[43] Q. Hu, C. W. Smith, N. F. Ness, and R. M. Skoug, J. Geophys. Res. 109, A03102 (2004).

[44] J. Zheng, Q. Hu, Y. Chen, and J. le Roux, J. Phys.: Conf. Ser. 900, 012024 (2017).

[45] J. T. Hoeksema et al., Solar Phys. 289, 3483 (2014). 